\title{
A Comprehensive Framework for Spectrum Sensing in Non-Linear and Generalized Fading Conditions
}

\author{
Paschalis C. Sofotasios ${ }^{(0)}$, Senior Member, IEEE, Alireza Bagheri, Student Member, IEEE, \\ Theodoros A. Tsiftsis ${ }^{(1)}$, Senior Member, IEEE, Steven Freear ${ }^{(0)}$, Senior Member, IEEE, Ali Shahzadi, \\ and Mikko Valkama, Senior Member, IEEE
}

\begin{abstract}
We derive a comprehensive analytical framework for the ED over generalized, extreme, and non-linear fading conditions which addresses the topic completely. This is carried out for both conventional and diversity receptions and it is based on the area under the ROC curve (AUC), which is an efficient performance measure that is widely used in physical sciences and engineering. This differentiates the considered methodology from the aforementioned routine approaches and additionally provides generic results on the arbitrary derivatives of the MGF of useful generalized processes. The asymptotic behavior of the derived expressions is also analyzed providing direct and concrete insights on the role and effect of the involved parameters on the ED performance. The offered analytic results are subsequently employed in quantifying the performance of ED over various types of fading conditions, which exhibits that ED performance is significantly degraded by even slight variations of the severity of fading. To this end, it is shown that the detrimental effects of fading can be effectively mitigated with the aid of square-law combining and switch-and-stay combining methods, as a low number of diversity branches can
\end{abstract}

Manuscript received April 10, 2016; revised October 22, 2016; accepted February 28, 2017. Date of publication April 25, 2017; date of current version October 13, 2017. This work was supported in part by the Academy of Finland, under Projects 284694 and 288670, and in part by the UK Engineering and Physical Sciences Research Council. This paper was presented in part at IEEE International Conference on Communications 2015, London, U.K., and at IEEE International Symposium on Personal, Indoor and Mobile Radio Communications, 2015, Hong Kong, [1]-[3]. The review of this paper was coordinated by Prof. C.-X. Wang. (Corresponding author: Paschalis C. Sofotasios.)

P. C. Sofotasios was with the School of Electronic and Electrical Engineering, University of Leeds, LS2 9JT Leeds, U.K. He is now with the Department of Electrical and Computer Engineering, Khalifa University of Science and Technology, Abu Dhabi 127788, UAE, and also with the Department of Electronics and Communications Engineering, Tampere University of Technology, 33101 Tampere, Finland (e-mail: p.sofotasios@ieee.org).

A. Bagheri was with the Department of Electrical and Computer Engineering, Semnan University, 35131-19111 Semnan, Iran. He is now with the Center for Wireless Communications and Signal Processing Research, Department of Electrical and Computer Engineering, New Jersey Institute of Technology, Newark, NJ 07102 USA (e-mail: ab745@njit.edu).

T. A. Tsiftsis is with the Department of Electrical Engineering, Technological Educational Institute of Central Greece, 35100 Lamia, Greece, and also with the Department of Electrical and Electronic Engineering, Nazarbayev University, Astana 010000, Kazakhstan (e-mail: tsiftsis@ teiste.gr).

$\mathrm{S}$. Freear is with the School of Electronic and Electrical Engineering, University of Leeds, LS2 9JT Leeds, U.K. (e-mail: s.freear@leeds.ac.uk).

A. Shahzadi is with the Department of Electrical and Computer Engineering, Semnan University, 35131-19111 Semnan, Iran (e-mail: shahzadi@ semnan.ac.ir).

M. Valkama is with the Department of Electronics and Communications Engineering, Tampere University of Technology, Tampere 33101, Finland (e-mail: mikko.e.valkama@tut.fi).

Color versions of one or more of the figures in this paper are available online at http://ieeexplore.ieee.org.

Digital Object Identifier 10.1109/TVT.2017.2692278 ensure sufficient and holistic performance improvement even in severe fading conditions.

Index Terms-Area under ROC curve, Bell polynomials, cognitive radio, diversity, multipath fading, radar systems.

\section{INTRODUCTION}

$\mathbf{T}$ HE detection of unknown wireless signals is considered an important topic in wireless communications and has for long attracted considerable attention by both academic and industrial sectors. It is also known that the efficiency of detectors is typically characterized by the following criteria: i) overall robustness; ii) no requirements of a priori knowledge of the characteristics of the signal under test (SUT); iii) operation at the shortest possible detection time; iv) low implementation complexity; v) ability to perform adequate detection over a wide range of frequency bands; vi) efficient operation in the presence of severe multipath fading and shadowing conditions [4]-[12] and the references therein. Based on these, different detection methods have been proposed for meeting certain performance and complexity requirements. These approaches have been developed in the context of energy detection, matched filter detection and cyclostationary, or feature detection [13], and achieving an adequate balance between high performance and low complexity constitutes a major challenge that becomes even more critical when accounting for realistic fading conditions of varying severity.

\section{A. Related Literature}

Matched filter-based detection is considered optimal thanks to the relatively low sensing time requirements. However, its main drawback is that its coherent structure requires a priori information about the features of the SUT, which is practically unrealistic in the majority of cognitive radio (CR) applications [14], [15]. Cyclostationary or feature detection methods exploit the inherent periodicity of modulated signals, which enables the detector to differentiate between the received signal and noise [16]. This approach provides adequate robustness but at a cost of high sampling rate and sensitivity of sampling clock errors, which renders it impractical in applications that require reduced computational complexity [13]. As a consequence, non-coherent detectors such as radiometers are widely employed thanks to their low computational and implementation 
complexities as well as the lack of requirements for a priori knowledge of the signal. As a result, even though ED appears vulnerable in the low signal-to-noise ratio (SNR) region and requires the noise power estimation process [17], [18], the aforementioned advantageous features constitute it the most suitable option in various applications relating to radar systems, impulse radio-based ultra-wideband systems, wireless sensor networks and spectrum sensing in CR systems [19]-[24].

It is recalled that the detection capability has been typically characterized by the probability of detection, $P_{d}$, and the probability of false alarm, $P_{f}$. These measures are fundamental as the former accounts for the probability that the presence of an unknown signal is detected, whereas the latter corresponds to the probability that a detector decides erroneously that an unknown signal is present. These measures are also used in forming the receiver operating characteristics (ROC) curve, $P_{d}$ versus $P_{f}$, for specific values of the time-bandwidth product and the energy threshold [25]. To this effect, detection of signals based on the received signal energy was firstly analyzed in [26] for the case of unknown deterministic signal over a flat band-limited Gaussian noise channel. Capitalizing on this, the ROC-based analysis of ED over Rayleigh, Nakagami-m, Rician, Weibull, $\eta-\mu, \kappa-\mu$ and $K, K_{G}$, respectively, were investigated in [27]-[33]. Likewise, similar analyses for different diversity methods were reported in [33]-[35], [37], [38] while a considerably improved energy detector was proposed in [39].

However, the conventional approach of ROC evaluation criteria imposes certain limitations when the considered fading conditions are generalized or composite. The main reason is that the involved mathematical complexity increases substantially, which renders the derivation of tractable closed-form expressions rather cumbersome, if not impossible. This is clearly reflected by the fact that most of the aforementioned analyses over generalized fading channels in [27]-[33] and [33]-[35], [37], [38] are based on either non-simple series representations or on semi-analytic approaches, which in turn exhibit a laborious and often problematic computation and further algebraic manipulation. In addition, although the ROC curves can provide a relatively adequate indication of ED performance, a single figure of merit that provides a better insight on the overall detection performance is undoubtedly useful as it can lead to more generic and reliable selection criteria. To this end, a distinct single-parameter measure that has been also used in various applications in natural sciences and engineering is the area under the ROC curve (AUC) [32]. This measure is an alternative metric for evaluating the performance of a detector and it has been used extensively in numerous areas of physical sciences and engineering. Its usefulness over the conventional $P_{f}$ and $P_{d}$ measures is that it constitutes a single-parameter measure that provides a better view of the overall detector performance. Specifically, in the conventional ROC approach the performance of spectrum sensing techniques depends on the value of the corresponding $P_{d}$ and $P_{f}$. Based on this, the most typical method to analyze the performance is to fix one of the two measures and vary the other one. This provides useful insights on the detector's performance which are, yet, specific and do not always extend readily to additionally account for the overall performance of the detector. On the contrary, in the case of AUC, there is only one parameter involved which provides a better insight on the overall performance. Ideally, AUC approaches unity, in which case the corresponding $P_{d}$ is always unity, regardless of the value of $P_{f}$. This is the main advantageous characteristic of AUC and useful values are considered those greater than 0.65 with those greater than 0.85 generally regarded as rather acceptable [40].

The AUC performance measure was firstly introduced in applications relating to wireless communications in [32], [33], [36]. Based on this, the authors in [38] analyzed the AUC by means of the semi-analytic moment-generating-function (MGF) approach for the case of Nakagami $-m$ and $\eta-\mu$ fading channels. Moreover, further addressing of similar topics based again on semi-analytic or non-explicit analyses were also reported in [34], [35], [41]. Nevertheless, following this approach has non-negligible drawbacks as the resulting analytic solutions are cumbersome to handle both analytically and numerically. Furthermore, a comprehensive mathematical framework over the most distinct generalized fading conditions does not exist neither for conventional nor for diversity based systems.

\section{B. Motivation and Contribution}

It is recalled that multipath fading can be accurately characterized by generalized statistical distributions. To this effect, the most generic and extensively used fading models are based on the $\kappa-\mu$, the $\eta-\mu$, and the $\alpha-\mu$ distributions, which were proposed in [42], [43]. These models are particularly flexible and have been proven capable of modeling effectively smallscale variations of the fading signal in line-of-sight (LOS) and non-line-of-sight (NLOS) conditions. This is also evident by the fact that they have been used in the derivation of accurate composite fading models, such as the recently proposed $\kappa-\mu$ shadowed model [44], [45]. In the same context, the $\kappa-\mu$ extreme distribution is a recently proposed fading model that provides accurate characterization of radio propagation under extreme fading channels, which are typically characterized by wireless communication scenarios that involve severe fading conditions and a small number of multipath components. Such scenarios are typically encountered in enclosed environments such as airplanes, train, buses and shopping malls [46].

However, in spite of the undoubted usefulness of the above models, their utilization in several applications is often limited due to their non-simple algebraic representation which renders the required analyses intractable. For example, evaluating the ED performance over $\alpha-\mu$ fading channels analytically is practically restricting even by the conventional ROC approach due to the arbitrary power of the argument of the involved exponential function. This also occurs for $\eta-\mu$ and $\kappa-\mu$ fading channels due to the presence of the modified Bessel function of the first kind. Motivated by this and based on the aforementioned advantages of the AUC metric, the present work is devoted to the derivation of a comprehensive mathematical framework for the ED over generalized fading conditions based on the AUC performance measure. As already mentioned, deriving such a framework in the context of conventional ROC approach is intractable and thus, the offered results are particularly useful in 
the comprehensive analysis of ED performance over various multipath fading conditions. Furthermore, the derivations are largely based on non-standard methods as the typically adopted semi-analytic MGF approach is extended in yielding elegant and tractable analytic solutions for both conventional and diversity based scenarios. In more details, the contributions of this paper are summarized below:

1) Novel and simple closed-form expressions are derived for the average AUC over $\kappa-\mu, \kappa-\mu$ extreme, $\eta-\mu$ and $\alpha-\mu$ fading channels.

2) The asymptotic behavior of the derived expressions is investigated thoroughly, allowing the development of direct insights on the impact of fading on ED performance.

3) The offered analytic results are extended to the case of square-law combining and switched diversity. This is realised by capitalizing on the semi-analytic method for deriving elegant analytic expressions. These diversity schemes do not require any channel state information (CSI) compared to schemes such as maximal ratio combining (MRC) and equal gain combining (EGC), which renders them suitable in practical ED-based systems.

4) The derived mathematical framework is employed in quantifying the effect of different fading conditions on the ED behavior and performance.

5) It is shown that the effect of the medium non-linearity on ED performance is the most significant while extreme fading conditions affect the ED performance dramatically.

6) It is shown that diversity reception can provide effective and robust compensation for the detrimental effects of generalized and extreme multipath fading. Indicatively, it is demonstrated that even a non-high number of branches can lead to acceptable ED in most fading conditions.

The remainder of the paper is organized as follows: The channel and system models are described in Section II. A novel mathematical framework for the average AUC of ED over $\kappa-\mu, \kappa-\mu$ extreme, $\eta-\mu$ and $\alpha-\mu$ fading channels is derived in Section III. This framework is extended to the case of square-law combining (SLC) and switched-and-stay combining (SSC) in Sections IV and V, respectively. Section VI presents the corresponding numerical results and useful discussions, while closing remarks are given in Section VII.

\section{System And Channel Models}

\section{A. System Model and Detection}

The problem of detecting the presence of unknown deterministic wireless signals can be modeled as a binary hypothesistesting problem, where hypotheses $H_{0}$ and $H_{1}$ correspond to the cases that the SUT is absent or present, respectively. To this effect, the received signal for the binary hypothesis is expressed as follows:

$$
r(t)=\left\{\begin{array}{cc}
n(t) & : H_{0} \\
a s(t)+n(t) & : H_{1}
\end{array}\right.
$$

where $a$ denotes a slow fading channel, $n(t)$ is the zero-mean complex additive white Gaussian noise (AWGN), with singlesided power spectral density $N_{0}$ at the receiver and $s(t)$ is the transmitted signal with average power $E_{s}$. The received signal is firstly band-pass filtered in bandwidth $W$ for removing the out of band noise and adjacent channel interference. This filter output is then absolute squared and integrated over time $T$, which yields the test statistic $Y$ that follows a central chisquare distribution, $\chi_{2 u}^{2}$, with $2 u$ degrees of freedom, in $H_{0}$ hypothesis and a non-central chi-square distribution, $\chi_{2 u}^{2}(2 \gamma)$, with $2 u$ degrees of freedom and a non-centrality parameter $2 \gamma$, in the $H_{1}$ hypothesis [4]. In these cases $u$ is the time-bandwidth product and

$$
\gamma=\frac{|a|^{2} E_{s}}{N_{0}}
$$

is the received instantaneous SNR. Finally, the test statistic $Y$ is compared with a predefined energy threshold $\lambda$, which determines whether the SUT is present or absent. To this effect, the probabilities of false alarm and detection are given by [4]

$$
\begin{aligned}
P_{f} & =\operatorname{Pr}\left(Y>\lambda \mid H_{0}\right) \\
& =\frac{\Gamma\left(u, \frac{\lambda}{2}\right)}{\Gamma(u)}
\end{aligned}
$$

and

$$
\begin{aligned}
P_{d} & =\operatorname{Pr}\left(Y>\lambda \mid H_{1}\right) \\
& =Q_{u}(\sqrt{2 \gamma}, \sqrt{\lambda})
\end{aligned}
$$

where $\Gamma(x)$ and $\Gamma(x, y)$ denote the gamma function and the upper incomplete gamma function, respectively, whereas $Q_{u}(a, b)$ is the generalized Marcum $Q$-function [47].

\section{B. Generalized Fading Channels}

It has already been mentioned that generalized fading models provide accurate characterization of multipath fading and include as special cases other commonly used distributions.

1) The $\kappa-\mu$ Distribution: This distribution was proposed as a generalized and versatile fading model that can provide accurate characterization of small-scale fading conditions also encountered in LOS communication scenarios. It has been proposed assuming that a signal is composed of clusters of multipath waves, propagating in a non-homogeneous environment. Within a cluster, the phases of the scattered waves are random and have similar delay times, while the delay-time spreads of different clusters are relatively large. Also, the clusters of multipath waves are assumed to have scattered waves with identical powers, but each cluster includes a dominant component with arbitrary power [42]. The parameter $\kappa$ denotes the ratio between the total power of the dominant components and the total power of the scattered waves, whereas $\mu$ is the number of multipath clusters.

The probability density function (PDF) of the instantaneous SNR per symbol, $\gamma$, is given by [42, eq. (10)]

$$
f_{\gamma}(\gamma)=\frac{\mu(1+\kappa)^{\frac{\mu+1}{2}}}{\kappa^{\frac{\mu-1}{2}} \exp (\kappa \mu)} \frac{\gamma^{\frac{\mu-1}{2}}}{\bar{\gamma}^{\frac{\mu+1}{2}}} \frac{I_{\mu-1}\left(2 \mu \sqrt{\frac{\kappa(1+\kappa) \gamma}{\bar{\gamma}}}\right)}{\exp \left(\frac{\mu(1+\kappa) \gamma}{\bar{\gamma}}\right)}
$$


where $\bar{\gamma}$ is the average SNR per symbol, and $I_{n}($.$) is the modi-$ fied Bessel function of the first kind [48]. It is recalled that the two fading parameters are linked to each other by

$$
\mu=\frac{1+2 \kappa}{\operatorname{Var}(\Omega)(1+\kappa)^{2}}
$$

with $\Omega$ and $\operatorname{Var}(\cdot)$ denoting the normalized power [42] and statistical variance, respectively. Furthermore, the $\kappa-\mu$ distribution includes as special cases Rice distribution for $\mu=1$, Nakagami $-m$ distribution for $\kappa \rightarrow 0$, Rayleigh distribution for $\mu=1$ and $\kappa \rightarrow 0$ and one-sided Gaussian for $\mu=0.5$ and $\kappa \rightarrow 0$, [42]. In addition, the respective MGF is expressed as [49, eq. (7)]

$$
M_{\gamma}(s)=\left(\frac{\mu(1+\kappa)}{s \bar{\gamma}+(1+\kappa) \mu}\right)^{\mu} \exp \left(-\frac{\mu s \kappa \bar{\gamma}}{s \bar{\gamma}+\mu(1+\kappa)}\right) .
$$

2) The $\kappa-\mu$ Extreme Distribution: This fading distribution was reported recently as an effective model for accounting for extreme fading conditions that are typically encountered in enclosed environments. This model emerged from $\kappa-\mu$ distribution, yet it is different, and assumes that $m$ remains constant while $\kappa$ and $\mu$ reach their extreme values, namely $\kappa \rightarrow \infty$, i.e. very strong LOS, and $\mu \rightarrow 0$, i.e. very few multipaths, [46]. The mentioned parameter $m$ is the inverse of the variance of the normalized power of the fading signal, i.e.,

$$
m=\frac{1}{\operatorname{Var}\left(P^{2}\right)}=\frac{1}{\operatorname{Var}(\Omega)}
$$

and is given in terms of $\kappa$ and $\mu$ as

$$
m=\frac{\mu(1+\kappa)^{2}}{1+2 \kappa} \text {. }
$$

This model also accounts for hyper-Rayleigh fading behavior [46] while its SNR PDF is defined as [29, eq. (22)], namely

$$
f_{\gamma}(\gamma)=\frac{2 m}{\sqrt{\gamma \bar{\gamma}}} e^{-2 m\left(1+\frac{\gamma}{\bar{\gamma}}\right)} I_{1}\left(4 m \sqrt{\frac{\gamma}{\bar{\gamma}}}\right)+\frac{e^{-2 m}}{2 \sqrt{\gamma \bar{\gamma}}} \delta(\gamma)
$$

where $\delta(\cdot)$ is the Dirac delta function. The algebraic representation of the $\kappa-\mu$ extreme distribution is relatively simple and its basic statistical metrics were derived in [46]. In what follows, we derive the MGF of the $\kappa-\mu$ extreme distribution which, among others, is useful in the subsequent analysis.

Theorem 1: The MGF of $\gamma$ for the $\kappa-\mu$ extreme distribution can be expressed as follows:

$$
M_{\gamma}(s)=e^{-\frac{2 m \bar{\gamma} s}{2 m+\bar{\gamma} s}}-e^{-2 m} .
$$

Proof: The proof is provided in Appendix I.

3) The $\eta-\mu$ Distribution: This distribution was proposed as a generalized and versatile fading model that can provide accurate characterization of small-scale fading conditions in NLOS communications. This fading model considers a signal composed of clusters of multipath waves propagating in a nonhomogeneous environment. Within any one cluster, the phases of the scattered waves are random and have similar delay times with the delay-time spreads of different clusters being relatively large [42]. The $\eta-\mu$ model consists of two formats with $\mu$ relating to the number of multipath clusters [42]. In Format I, $0<\eta<\infty$ is the ratio between the in-phase and quadrature components of the fading signal within each cluster. These components are assumed to be statistically independent to each other and have different powers. In Format II, $-1<\eta<1$ is the correlation between the powers of the in-phase and quadrature scattered waves in each multipath cluster. The corresponding SNR PDF is given by [42, eq. (26)]

$$
f_{\gamma}(\gamma)=\frac{2 \sqrt{\pi} h^{\mu} \mu^{\mu+\frac{1}{2}} \gamma^{\mu-\frac{1}{2}}}{\Gamma(\mu) H^{\mu-\frac{1}{2}} \bar{\gamma}^{\mu+\frac{1}{2}}} e^{-\frac{2 \mu h}{\gamma} \gamma} I_{\mu-\frac{1}{2}}\left(\frac{2 \mu H}{\bar{\gamma}} \gamma\right)
$$

where

$$
\begin{aligned}
h & =\frac{2+\eta^{-1}+\eta}{4}, \\
H & =\frac{\eta^{-1}-\eta}{4}
\end{aligned}
$$

in Format I and

$$
\begin{aligned}
h & =\frac{1}{1-\eta^{2}}, \\
H & =\frac{\eta}{1-\eta^{2}}
\end{aligned}
$$

in Format II. It is noted here that the $\mu$ parameter in the $\eta-\mu$ fading model denotes half the number of multipath clusters and is linked with $\eta$ by [42]

$$
\mu=\frac{1+(H / h)^{2}}{2 \operatorname{Var}(\Omega)} .
$$

Furthermore, the $\eta-\mu$ distribution reduces to Nakagami $-q$ (Hoyt) distribution for $\mu=0.5$ and to Nakagami $-m$ distribution for $\eta \rightarrow 0, \eta \rightarrow \infty$, and $\eta \rightarrow \pm 1$. Also, the MGF of $\gamma$ for the $\eta-\mu$ fading distribution is expressed as [49, eq. (6)]

$$
M_{\gamma}(s)=\left(\frac{4 \mu^{2} h}{(2(h-H) \mu+\bar{\gamma} s)(2(h+H) \mu+\bar{\gamma} s)}\right)^{\mu} .
$$

4) The $\alpha-\mu$ Distribution: This distribution was proposed as a generalized model that can provide accurate characterization of small-scale fading conditions by also taking into account the non-linearity of the propagation medium. The $\alpha-\mu$ fading model considers a signal composed of clusters of multipath waves propagating in a non-homogeneous environment. Within any one cluster, the phases of the scattered waves are random and have similar delay times, while delay-time spreads of different clusters are relatively large. These clusters are assumed to consist of scattered waves with identical powers and the resulting envelope is obtained as a nonlinear function of the modulus of the sum of the multipath components. Such a nonlinearity is manifested in terms of a power parameter, so that the resulting signal intensity is obtained not only as the modulus of the sum of the multipath components, but as the modulus to a certain given exponent [43]. The corresponding SNR PDF is given by [43, eq. (1)]

$$
f_{\gamma}(\gamma)=\frac{\alpha \mu^{\mu}}{2 \Gamma(\mu) \bar{\gamma}^{\frac{\alpha \mu}{2}}} \gamma^{\frac{\alpha \mu}{2}-1} \exp \left(-\mu \frac{\gamma^{\alpha / 2}}{\bar{\gamma}^{\alpha / 2}}\right)
$$


where $\mu$ is the number of multipath clusters and $\alpha>0$ denotes the non-linearity of the medium, which is subsequently shown to be critical in ED performance. This model reduces to Weibull model for $\mu=1$, to Nakagami $-m$ model for $\alpha=2$ and to Rayleigh model for $\alpha=2$ and $\mu=1$. Also, the corresponding MGF of $\gamma$ is given by [50, eq. (6)] in (22), as shown bottom of this page,

$$
\begin{aligned}
M_{\gamma}(s)= & \frac{\alpha \mu^{\mu}}{2 \Gamma(\mu) \bar{\gamma}^{\frac{\alpha \mu}{2}}} \frac{k^{\frac{1}{2}} l^{\frac{\alpha \mu-1}{2}}}{(2 \pi)^{\frac{l+k-2}{2}} s^{\frac{\alpha \mu}{2}}} \\
& \times G_{l, k}^{k, l}\left(\left(\frac{\mu}{\bar{\gamma}^{\frac{\alpha}{2}}}\right)^{k} \frac{l^{l}}{k^{k} s^{l}} \mid \begin{array}{c}
\Delta\left(l, 1-\frac{\alpha \mu}{2}\right) \\
\Delta(k, 0)
\end{array}\right)
\end{aligned}
$$

where $G_{p, q}^{m, n}($.$) is the Meijer G$-function [48, eq. (9.301)] and

$$
\Delta(a, b)=\frac{b}{a}, \frac{b+1}{a}, \ldots, \frac{b+a-1}{a} .
$$

\section{Area Under ROC Curve (AUC)}

As already mentioned, the AUC is an effective singleparameter performance measure that has been used extensively in various analyses in natural sciences and engineering. The AUC is the area covered by the ROC curve of $P_{d}$ versus $P_{f}$ and is defined as [25], [32]

$$
A=\int_{0}^{1} P_{d}(\gamma, \lambda) \mathrm{d} P_{f}(\lambda)
$$

Thus, the average AUC $(\overline{\mathrm{AUC}})$ is determined by averaging (24) over the statistics of the fading conditions, namely

$$
\overline{\mathrm{AUC}}=\int_{0}^{\infty} A(\gamma) f_{\gamma}(\gamma) \mathrm{d} \gamma
$$

The AUC over AWGN channels is expressed as [41, eq. (12)]

$$
A(\gamma)=1-\sum_{l=0}^{u-1} \sum_{i=0}^{l}\left(\begin{array}{c}
l+u-1 \\
l-i
\end{array}\right) \frac{\gamma^{i} e^{-\frac{\gamma}{2}}}{i ! 2^{l+i+u}}
$$

for integer values of $u$, with $\left(\begin{array}{l}a \\ b\end{array}\right)$ denoting the binomial coefficient. Likewise, in the presence of fading effects, the average AUC is obtained by substituting (26) in (25) yielding

$\overline{\mathrm{AUC}}=1-\sum_{l=0}^{u-1} \sum_{i=0}^{l}\left(\begin{array}{c}l+u-1 \\ l-i\end{array}\right)\left(\frac{1}{2}\right)^{l+i+u} \int_{0}^{\infty} \frac{\gamma^{i} f_{\gamma}(\gamma)}{i ! e^{\frac{\gamma}{2}}} \mathrm{~d} \gamma$

as well as by the following MGF based semi-analytic approach

$$
\overline{\mathrm{AUC}}=1-\left.\sum_{l=0}^{u-1} \sum_{i=0}^{l}\left(\begin{array}{c}
u+l-1 \\
l-i
\end{array}\right) \frac{(-1)^{i}}{i ! 2^{u+l+i}} M_{\gamma}^{(i)}(s)\right|_{s=\frac{1}{2}}
$$

where $M_{\gamma}^{(n)}(s)$ denotes the $n$th derivative of the MGF of the fading involved distribution. It is noted that (24)-(28) can be also expressed in terms of the complementary AUC, namely $\mathrm{CAUC}=1-\mathrm{AUC}$. Furthermore, in the subsequent analysis we capitalize (28) to derive analytic expressions which are not expressed in semi-analytic form, such as the derivation of (13) in Theorem 1. Hence, the adopted method is different and more efficient than the typical semi-analytic MGF approach.

\section{Average AUC OVER GENERALIZED AND Non-LinEAR FADING CHANNELS}

This section is devoted to the derivation of a mathematical framework for the average AUC over generalized and non-linear fading channels for single channel communications.

\section{A. Average AUC over $\kappa-\mu$ Fading Channels}

Theorem 2: For $\kappa, \mu, \bar{\gamma} \in \mathbb{R}^{+}$and $u \in \mathbb{N}$, the average AUC over $\kappa-\mu$ fading channels can be expressed as follows:

$$
\begin{gathered}
\overline{\mathrm{AUC}}_{\kappa-\mu}=1-c_{1} \sum_{l=0}^{u-1} \sum_{i=0}^{l} \sum_{j=0}^{i}\left(\begin{array}{c}
l+u-1 \\
l-i
\end{array}\right)\left(\begin{array}{c}
i+\mu-1 \\
i-j
\end{array}\right) \\
\times \frac{1}{2^{l} j !}\left(\frac{1}{1+2 \zeta}\right)^{i}\left(\frac{2 \kappa \mu \zeta}{1+2 \zeta}\right)^{j}
\end{gathered}
$$

where $\zeta=\frac{\mu(1+\kappa)}{\bar{\gamma}}$ and

$$
c_{1}=\frac{1}{2^{u}}\left(\frac{2 \zeta}{1+2 \zeta}\right)^{\mu} e^{-\frac{\kappa \mu}{1+2 \zeta}}
$$

Proof: The proof is provided at Appendix II.

Importantly, the derived expression in (29) consists only of elementary functions, which renders it simple as it can be readily computed using popular mathematical software packages such as MATLAB, MAPLE and MATHEMATICA. Furthermore, it can be further analyzed asymptotically leading to useful insights on the impact of the involved parameters on the system performance. To this end, in the case of high SNR and not particularly strong LOS components it is realistic to assume that $\bar{\gamma}>>2 \mu(1+\kappa)$; based on this (29) becomes

$$
\begin{aligned}
\overline{\mathrm{AUC}}_{\kappa-\mu}^{\mathrm{asym}}{ }^{+} \simeq 1- & \frac{e^{-\frac{\kappa \mu}{\gamma}}}{2^{u} \bar{\gamma}^{\mu}} \sum_{l=0}^{u-1} \sum_{i=0}^{l} \sum_{j=0}^{i}\left(\begin{array}{c}
l+u-1 \\
l-i
\end{array}\right) \\
& \times\left(\begin{array}{c}
i+\mu-1 \\
i-j
\end{array}\right) \frac{1}{2^{l} j !}\left(\frac{2 \kappa \mu^{2}(1+\kappa)}{\bar{\gamma}}\right)^{j} .
\end{aligned}
$$

By also noticing that the above triple series is dominated by the fraction outside of it, one obtains

$$
\overline{\mathrm{AUC}}_{\kappa-\mu}^{\mathrm{asym}^{+}} \propto 1-2^{-u} \bar{\gamma}^{-\mu} e^{-\frac{\kappa \mu}{\bar{\gamma}}}
$$

The above expression shows clearly that the effect of $\kappa$ and $\mu$ are exponential. As a result, in case of strong LOS components the involved fraction reduces rapidly to zero and thus, $\overline{\mathrm{AUC}}_{\kappa-\mu}^{\mathrm{asym}^{+}} \rightarrow 1$. Likewise, for the crucial case of low SNR values it is realistic to assume that $\bar{\gamma}<<2 \mu(1+\kappa)$. By substituting in (29) and carrying out some long but basic algebraic 
manipulations one obtains

$$
\begin{aligned}
& \overline{\mathrm{AUC}}_{\kappa-\mu}^{\mathrm{asym}^{-}} \simeq 1-\frac{e^{-\frac{\kappa \bar{\gamma}}{2(1+\kappa)}}}{2^{u}} \sum_{l=0}^{u-1} \sum_{i=0}^{l} \sum_{j=0}^{i}\left(\begin{array}{c}
l+u-1 \\
l-i
\end{array}\right) \\
& \times\left(\begin{array}{c}
i+\mu-1 \\
i-j
\end{array}\right) \frac{1}{2^{l} j !}\left(\frac{\bar{\gamma}}{2 \mu(1+\kappa)}\right)^{i}(\kappa \mu)^{j}
\end{aligned}
$$

which for low values of $\bar{\gamma}$ reduces to

$$
\overline{\mathrm{AUC}}_{\kappa-\mu}^{\operatorname{asym}^{-}} \propto 1-2^{-u} e^{-\frac{\kappa \bar{\gamma}}{2(1+\kappa)}}
$$

which is rather similar to (32). Nevertheless, eq. (34) indicates that the effect of $\mu$ on $\overline{\mathrm{AUC}}$ is negligible in the low SNR regime. Also, the effect of $\kappa$ is relatively quick even for a clear LOS component while the time-bandwidth product is the parameter that can practically improve the value of $\overline{\mathrm{AUC}}$.

\section{B. Average AUC over $\kappa-\mu$ Extreme Fading Channels}

Theorem 3: For $m, \bar{\gamma} \in \mathbb{R}^{+}$and $u \in \mathbb{N}$, the average AUC over $\kappa-\mu$ extreme fading channels can be expressed as follows:

$$
\begin{aligned}
\overline{\mathrm{AUC}}_{\kappa-\mu \text { Ext. }}=1-\sum_{l=0}^{u-1} & \sum_{i=0}^{l}\left(\begin{array}{c}
l+u-1 \\
l-i
\end{array}\right) \frac{8 m^{2} e^{-2 m} \bar{\gamma}^{i}}{2^{l+u}(\bar{\gamma}+4 m)^{i+1}} \\
& \times{ }_{1} F_{1}\left(i+1 ; 2 ; \frac{8 m^{2}}{\bar{\gamma}+4 m}\right) \cdot
\end{aligned}
$$

Proof: The proof is provided in Appendix III.

Equation (35) has also simple algebraic representation as the Kummer's hypergeometric function is built-in in all popular mathematical software packages and its monotonicity properties are well studied. Thus, for the practical scenarios of $\bar{\gamma}>20 \mathrm{~dB}$, it is realistic to assume that $\bar{\gamma}+4 m \approx \bar{\gamma}$ yielding

$$
\begin{gathered}
\overline{\mathrm{AUC}}_{\kappa-\mu \mathrm{Ext} .}^{\mathrm{asym}^{+}} \simeq 1-\frac{m^{2} e^{-2 m}}{2^{u-3} \bar{\gamma}} \sum_{l=0}^{u-1} \sum_{i=0}^{l}\left(\begin{array}{c}
l+u-1 \\
l-i
\end{array}\right) \frac{1}{2^{l}} \\
\times{ }_{1} F_{1}\left(i+1 ; 2 ; \frac{8 m^{2}}{\bar{\gamma}}\right) .
\end{gathered}
$$

By recalling that for high values of $\bar{\gamma}$ the argument of the ${ }_{1} F_{1}(\cdot)$ function tends to zero and also noticing that the double summation is dominated by the terms outside of it yields

$$
\overline{\mathrm{AUC}}_{\kappa-\mu \text { Ext. }}^{\mathrm{asym}+} \propto 1-2^{3-u} \bar{\gamma}^{-1} e^{-2 m}
$$

which shows the impact of $\bar{\gamma}, u$ and $m$ in the average AUC when the second term in (37) tends to zero, i.e. the $\overline{\mathrm{AUC}}$ approaches unity. More importantly, for the case of $\bar{\gamma}<-10 \mathrm{~dB}$, it follows that $\bar{\gamma}<<4 \mathrm{~m}$, which yields

$\overline{\mathrm{AUC}}_{\kappa-\mu \text { Ext. }}^{\mathrm{asym}^{-}} \simeq 1-\sum_{l=0}^{u-1} \sum_{i=0}^{l}\left(\begin{array}{c}l+u-1 \\ l-i\end{array}\right) \frac{\bar{\gamma}^{i}{ }_{1} F_{1}(i+1 ; 2 ; 2 m)}{2^{2 i+l+u-1} m^{i-1} e^{2 m}}$.

Given that the exponential term dominates in the above expression, it immediately follows that

$$
\overline{\mathrm{AUC}}_{\kappa-\mu \text { Ext. }}^{\mathrm{asym}^{-}} \propto 2^{1-u} e^{-2 m}
$$

which shows that the value of $\overline{\mathrm{AUC}}$ in the low average SNR regime depends on the value of the time-bandwidth product as well as on the severity of the encountered fading conditions.

\section{Average AUC over $\eta-\mu$ Fading Channels}

Theorem 4: For $\bar{\gamma}, \mu, h, H \in \mathbb{R}^{+}$and $u \in \mathbb{N}$, the following closed-form representation is valid for the average AUC over $\eta-\mu$ fading channels

$$
\begin{aligned}
\overline{\mathrm{AUC}}_{\eta-\mu}=1-c_{2} & \sum_{l=0}^{u-1} \sum_{i=0}^{l}\left(\begin{array}{c}
l+u-1 \\
l-i
\end{array}\right)\left(\begin{array}{c}
i+2 \mu-1 \\
i
\end{array}\right) \\
& \times \frac{{ }_{2} F_{1}\left(\frac{i+2 \mu}{2}, \frac{i+2 \mu+1}{2}, \mu+\frac{1}{2} ; \frac{16 \mu^{2} H^{2}}{(\bar{\gamma}+4 \mu h)^{2}}\right)}{2^{l} \bar{\gamma}^{-i}(\bar{\gamma}+4 \mu h)^{i}}
\end{aligned}
$$

where ${ }_{2} F_{1}(\cdot)$ denotes the Gauss hypergeometric function and

$$
c_{2}=\frac{1}{2^{u}}\left(\frac{4 \mu \sqrt{h}}{\bar{\gamma}+4 \mu h}\right)^{2 \mu}
$$

Proof: The proof is provided in Appendix IV.

Equation (40) has a similar algebraic representation to (35), which allows the development of insights on the asymptotic behavior at high and low SNR regimes. To this end, when $\bar{\gamma}>15$ $\mathrm{dB}$ we can realistically assume that $\bar{\gamma}>>4 \mu h$, yielding

$$
\begin{aligned}
\overline{\mathrm{AUC}}_{\eta-\mu}^{\mathrm{asym}}{ }^{+} \simeq 1-\sum_{l=0}^{u-1} & \sum_{i=0}^{l}\left(\begin{array}{c}
l+u-1 \\
l-i
\end{array}\right)\left(\begin{array}{c}
i+2 \mu-1 \\
i
\end{array}\right) \\
& \times \frac{{ }_{2} F_{1}\left(\frac{i+2 \mu}{2}, \frac{i+2 \mu+1}{2}, \mu+\frac{1}{2} ; \frac{16 \mu^{2} H^{2}}{\bar{\gamma}}\right)}{2^{u} \bar{\gamma}^{2 \mu}}
\end{aligned}
$$

where for high SNR values, $16 \mu^{2} H^{2} / \bar{\gamma} \approx 0$, which results to ${ }_{2} F_{1}(\cdot) \approx 1$. Based on this, it immediately follows that

$$
\overline{\mathrm{AUC}}_{\eta-\mu}^{\mathrm{asym}}{ }^{+} \propto 1-2^{-u} \bar{\gamma}^{-2 \mu}
$$

which shows that the effect of $\eta$ becomes negligible at the high SNR regime, where $\overline{\mathrm{AUC}}$ depends only on the value of $u$ and $\mu$. Thus, for small values of $u$ and $\mu$ the $1 / 2^{u} \bar{\gamma}^{2 \mu}$ term does not reduce to zero and $\overline{\mathrm{AUC}}$ is less than unity. Likewise, for the case of low SNR values i.e $\bar{\gamma}<10 \mathrm{~dB}$, we can accurately assume that $\bar{\gamma}<<4 \mu h$. As a result (40) can be re-written as

$$
\begin{aligned}
\overline{\mathrm{AUC}}_{\eta-\mu}^{\mathrm{asym}^{-}} \simeq 1-\sum_{l=0}^{u-1} & \sum_{i=0}^{l}\left(\begin{array}{c}
l+u-1 \\
l-i
\end{array}\right)\left(\begin{array}{c}
i+2 \mu-1 \\
i
\end{array}\right) \\
\times & \frac{\bar{\gamma}^{i}{ }_{2} F_{1}\left(\frac{i+2 \mu}{2}, \frac{i+2 \mu+1}{2}, \mu+\frac{1}{2} ; \frac{H^{2}}{h^{2}}\right)}{2^{u+l+2 i} h^{\mu+i} \mu^{i}} .
\end{aligned}
$$

Evidently, the range of values of the above triple series is rather small; based on this, one obtains

$$
\overline{\mathrm{AUC}}_{\eta-\mu}^{\operatorname{asym}^{-}} \propto 1-2^{-u} h^{-\mu}
$$


which according to the two formats of the $\eta-\mu$ distribution can be equivalently expressed as

$$
\overline{\mathrm{AUC}}_{\eta-\mu}^{\mathrm{asym}^{-}} \propto 1-\frac{2^{2 \mu} \eta^{\mu}}{2^{u}\left(1-\eta^{2}\right)^{\mu}}
$$

for Format I and

$$
\overline{\mathrm{AUC}}_{\eta-\mu}^{\mathrm{asym}^{-}} \propto 1-\left(1-\eta^{2}\right)^{\mu} 2^{-u}
$$

for Format II, respectively. By recalling the physical interpretation of $\eta$, the above relationships exhibit that a satisfactory $\overline{\mathrm{AUC}}$ in the low SNR regime can be achieved when the ratio or correlation of the powers of the in-phase and quadrature components is low and the time-bandwidth product is rather high, so that the second terms of (46)-(47) reduce to zero.

\section{Average AUC over $\alpha-\mu$ Fading Channels}

Theorem 5: For $\alpha, \mu, \bar{\gamma} \in \mathbb{R}^{+}$and $u \in \mathbb{N}$, the average AUC over $\alpha-\mu$ fading channels can be represented as follows:

$$
\begin{aligned}
& \overline{\mathrm{AUC}}_{\alpha-\mu}=1- c_{3} \sum_{i=0}^{u-1} \sum_{j=0}^{i}\left(\begin{array}{c}
i+u-1 \\
i-j
\end{array}\right) \frac{\alpha^{j}}{2^{i} j !} \\
& \times G_{\alpha, 2}^{2, \alpha}\left(\frac{\mu^{2} 2^{\alpha} \alpha^{\alpha}}{4 \bar{\gamma}^{\alpha}} \mid \begin{array}{c}
\Delta\left(\begin{array}{c}
\left.\alpha, j-j-\frac{\alpha \mu}{2}\right) \\
\Delta(2,0)
\end{array}\right)
\end{array}\right.
\end{aligned}
$$

where

$$
c_{3}=\frac{\mu^{\mu} \alpha^{\frac{\alpha \mu+1}{2}}}{2^{u+\frac{\alpha(1-\mu)+1}{2}} \pi^{\frac{\alpha}{2}} \bar{\gamma}^{\frac{\alpha \mu}{2}} \Gamma(\mu)} .
$$

Proof: The proof is provided in Appendix V.

Equation (48) is expressed in closed-form and can be numerically evaluated with the aid of popular software packages that include the Meijer $G$-function as a built-in function. By also recalling the monotonicity properties of this highly non-linear function, it is shown that in the high SNR regime the $\bar{\gamma}$ term in the argument of the Meiger $G$-function becomes dominant and thus, the function and the whole double series are practically negligible. Based on this, it follows that

$$
\overline{\mathrm{AUC}}_{\eta-\mu}^{\mathrm{asym}^{+}} \simeq 1-\frac{\mu^{\mu} \alpha^{\frac{\alpha \mu+1}{2}}}{2^{u+\frac{\alpha(1-\mu)+1}{2}} \pi^{\frac{\alpha}{2}} \bar{\gamma}^{\frac{\alpha \mu}{2}} \Gamma(\mu)}
$$

which yields

$$
\overline{\mathrm{AUC}}_{\eta-\mu}^{\mathrm{asym}^{+}} \propto 1-\frac{1}{2^{u+\frac{\alpha(1-\mu)+1}{2}} \pi^{\frac{\alpha}{2}} \bar{\gamma}^{\frac{\alpha \mu}{2}} \Gamma(\mu)} .
$$

It is evident that for high values of $\bar{\gamma}, \alpha$ and $\mu$, the second term in (51) reduces rapidly to zero and the energy detector tends to achieve ideal performance as $\overline{\mathrm{AUC}}_{\eta-\mu}^{\mathrm{asym}^{+}} \rightarrow 1$.

\section{AVERAge AUC ANALYSIS With SLC Diversity RECEPTIONS}

The derived analytic results in the previous section can be extended to cases of diversity reception, which are capable of providing significant improvement of the detection performance. In the case of SLC method, $L$ diversity branches are combined upon squaring and integration of the received signal. Hence, the output SNR in SLC is the sum of the SNRs in the involved branches i.e.

$$
\gamma_{\mathrm{SLC}}=\sum_{i=1}^{L} \gamma_{i} .
$$

Thus, for the case of independent and non-identically distributed branches, the corresponding MGF is given by [59]

$$
M_{\gamma_{\mathrm{SLC}}}(s)=\prod_{i=1}^{L} M_{\gamma, i}(s) .
$$

Based on this and with the aid of the Leibniz's differential product rule in [48, eq. $(0.42)]$, it follows that

$$
(f \cdot h)^{(n)}=\sum_{k=0}^{n}\left(\begin{array}{l}
n \\
k
\end{array}\right) f^{(k)} h^{(n-k)}
$$

which is a rather useful and relatively simple finite series representation. To this effect, the average AUC over fading channels using SLC can be generically expressed by (55), as shown the bottom of this page. This expression has been used in several analyses in ED based spectrum sensing; yet, its main drawback is that it is semi-analytic as it requires evaluation of the $n$th derivative of the MGF of the considered fading model. In what follows, we capitalize on the above standard method and derive exact closed-form expressions for the $n$th derivative of the MGF for $\kappa-\mu, \kappa-\mu$ extreme, $\eta-\mu$ and $\alpha-\mu$ fading channels. The derived results are novel, generic and elegant while unlike the cumbersome and laborious conventional semi-analytic approach, they can be computed rather straightforwardly. In addition, the usefulness of the offered results is evident by the fact that besides their importance in the present analyses, they can be also useful in numerous other investigations, including several applications in wireless communications, such as those in [53]-[56].

\section{A. Average AUC over $\kappa-\mu$ Fading Channels}

Lemma 1: For $\kappa, \mu, \bar{\gamma}, s \in \mathbb{R}^{+}$and $n \in \mathbb{N}$, the following generic closed-form expression is valid for the $n$th derivative

$$
\begin{aligned}
\overline{\mathrm{AUC}}^{\mathrm{SLC}}= & 1-\sum_{l=0}^{u-1} \sum_{i=0}^{l}\left(\begin{array}{c}
l+u-1 \\
l-i
\end{array}\right)\left(\frac{1}{2}\right)^{l+i+u} \frac{(-1)^{i}}{i !} \sum_{j_{1}=0}^{i} \sum_{j_{2}=0}^{j_{1}} \cdots \sum_{j_{L-1}=0}^{j_{L-2}}\left(\begin{array}{c}
i \\
j_{1}
\end{array}\right)\left(\begin{array}{l}
j_{1} \\
j_{2}
\end{array}\right) \cdots \\
& \times\left.\left(\begin{array}{c}
j_{L-2} \\
j_{L-1}
\end{array}\right) M_{\gamma, 1}^{\left(i-j_{1}\right)}(s) M_{\gamma, 2}^{\left(j_{1}-j_{2}\right)}(s) \cdots M_{\gamma, L-1}^{\left(j_{L-2}-j_{L-1}\right)}(s) M_{\gamma, L}^{\left(j_{L-1}\right)}(s)\right|_{s=\frac{1}{2} .}
\end{aligned}
$$


of the moment-generating-function of the $\kappa-\mu$ distribution

$$
\begin{aligned}
M_{\kappa-\mu}^{(n)}(s)=M_{\gamma} & (s) \sum_{i=0}^{n}\left(\begin{array}{c}
n \\
i
\end{array}\right)(\mu)_{(n-i)} B_{i} \\
& \times\left(-\frac{\bar{\gamma}}{\bar{\gamma} s+(1+\kappa) \mu}\right)^{n-i}
\end{aligned}
$$

where $(x)_{n}$ denotes the Pochhammer symbol and

$$
B_{n}\left(x_{1}, \ldots, x_{n}\right) \triangleq \sum_{k=1}^{n} B_{n, k}\left(x_{1}, \ldots, x_{n-k+1}\right)
$$

is the $n$th complete Bell polynomials with $B_{n, k}$ denoting the partial Bell polynomials [53], [57].

Proof: The proof is provided in Appendix VI.

Equation (56) has a simple algebraic form and can be readily computed because the $B_{n, k}$ function is built-in popular mathematical software packages, such as MATHEMATICA.

\section{B. Average AUC over $\kappa-\mu$ Extreme Fading Channels}

Lemma 2: For $m, \bar{\gamma}, s \in \mathbb{R}^{+}$and $n \in \mathbb{N}$, the following generic closed-form expression is valid for the $n$th derivative of the moment-generating-function of the $\kappa-\mu$ extreme distribution

$$
M_{\kappa-\mu \text { Ext. }}^{(n)}(s)=e^{-\frac{2 m \bar{\gamma} s}{2 m+\bar{\gamma} s}} B_{n}
$$

Proof: We let

$$
M_{\gamma}(s)=e^{g(s)}-e^{-2 m}
$$

with

$$
g(s)=-\frac{2 m \bar{\gamma} s}{2 m+\bar{\gamma} s} .
$$

Based on this and considering this fact that the second term is a constant value yields (58), which completes the proof.

\section{Average AUC over $\eta-\mu$ Fading Channels}

Lemma 3: For $h, H, \mu, \bar{\gamma}, \in \mathbb{R}^{+}$and $n \in \mathbb{N}$, the following closed-form expression is valid for the $n$th derivative of the moment-generating-function of the $\eta-\mu$ fading distribution

$$
\begin{aligned}
M_{\eta-\mu}^{(n)}(s)=\sum_{i=0}^{n}\left(\begin{array}{l}
n \\
i
\end{array}\right) \frac{(-1)^{n}\left(4 \mu^{2} h\right)^{\mu}(\mu)_{i}(\mu)_{n-i} \bar{\gamma}^{n}}{(2(h-H) \mu+\bar{\gamma} s)^{\mu+i}} \\
\times \frac{1}{(2(h+H) \mu+\bar{\gamma} s)^{\mu+n-i}} .
\end{aligned}
$$

Proof: The proof is provided in Appendix VII.

The above expressions are significantly more tractable than those obtained by the conventional and often used semi-analytic approach. This renders them analytically compact and straightforward to compute because the involved functions are included in all major mathematical software packages.

Finally, a closed-form expression is derived for the MGF of the $\alpha-\mu$ fading distribution.

\section{Average AUC over $\alpha-\mu$ Fading Channels}

Lemma 4: For $\alpha, \mu, \bar{\gamma}, s \in \mathbb{R}^{+}$and $n, l, k \in \mathbb{N}$ with $l / k=$ $\alpha / 2$, the following closed-form expression holds for the $n$th derivative of the moment-generating-function of the $\alpha-\mu$ distribution

$$
\begin{aligned}
M_{\alpha-\mu}^{(n)} & (s)=c_{4} \sum_{i=0}^{n}\left(\begin{array}{c}
n \\
i
\end{array}\right) \frac{(-1)^{n-i} l^{i}\left(\frac{\alpha \mu}{2}\right)_{n-i}}{s^{\frac{\alpha \mu}{2}+n}} \\
& \times G_{2 l, k+l}^{k+l, l}\left(\frac{\mu^{k} l^{l}}{\bar{\gamma}^{\frac{\alpha k}{2}} k^{k} s^{l}} \mid \begin{array}{c}
\Delta\left(l, 1-\frac{\alpha \mu}{2}\right), \Delta(l, 1-i) \\
\Delta(l, 1), \Delta(k, 0)
\end{array}\right)
\end{aligned}
$$

where

$$
c_{4}=\frac{\alpha \mu^{\mu} k^{\frac{1}{2}} l^{\frac{\alpha \mu-1}{2}}}{2 \Gamma(\mu) \bar{\gamma}^{\frac{\alpha \mu}{2}}(2 \pi)^{\frac{l+k-2}{2}}} .
$$

Proof: The proof is provided in Appendix VIII.

The $n$th derivative of the above MGF is less simple compared to those for the $\kappa-\mu, \kappa-\mu$ extreme and $\eta-\mu$ distributions due to the presence of the highly non-linear Meijer $G$-function. Yet, its representation is given in closed-form and its computation is more simple than the conventional semi-analytic MGF approach in (55). The MGF and its $n$th derivative for each of the aforementioned models are illustrated in summary in Table I. Hence, it is evident that exact closed-form expressions can be readily deduced for the average AUC over $\kappa-\mu, \kappa-\mu$ extreme, $\eta-\mu$ and $\alpha-\mu$ fading channels by applying Lemmas $1-4$ in (55).

\section{AVERAge AUC With Switched Diversity RECEPTION}

Switched diversity is a relatively simple and effective diversity method. Let $\gamma_{\mathrm{SSC}}$ and $\gamma_{T}$ denote the SNR per symbol of the SSC combiner output and the predefined switching threshold, respectively, the PDF in the case of dual branch is expressed as [54]

$$
f_{\gamma_{\mathrm{SSC}}}(\gamma)=\left\{\begin{array}{cc}
F_{\gamma}\left(\gamma_{T}\right) f_{\gamma}(\gamma) & \gamma<\gamma_{T} \\
\left(1+F_{\gamma}\left(\gamma_{T}\right)\right) f_{\gamma}(\gamma) & \gamma \geq \gamma_{T}
\end{array}\right.
$$

where $F_{\gamma}(\cdot)$ denotes the respective cumulative distribution function (CDF). Hence, the average AUC for the dual SSC diversity scheme is obtained by averaging (24) over (64), which yields

$$
\overline{\mathrm{AUC}}^{\mathrm{SSC}}=F_{\gamma}\left(\gamma_{T}\right) \overline{\mathrm{AUC}}+\int_{\gamma_{T}}^{\infty} A(\gamma) f_{\gamma}(\gamma) \mathrm{d} \gamma
$$

To this effect and with the aid of (26), the average AUC over fading channels for the case of integer values of $u$ can be comprehensively expressed by the following generic representation

$$
\begin{aligned}
\overline{\mathrm{AUC}}_{\mathrm{SSC}}=1-F_{\gamma}\left(\gamma_{T}\right) \overline{\mathrm{CAUC}}-\sum_{l=0}^{u-1} \sum_{i=0}^{l}\left(\begin{array}{c}
l+u-1 \\
l-i
\end{array}\right) \\
\times\left(\frac{1}{2}\right)^{l+i+u} \frac{1}{i !} \int_{\gamma_{T}}^{\infty} \gamma^{i} e^{-\frac{\gamma}{2}} f_{\gamma}(\gamma) \mathrm{d} \gamma .
\end{aligned}
$$

Next, we utilize (66) in deriving novel exact closed-form expressions for the average AUC using SSC under $\kappa-\mu, \kappa-\mu$ extreme, $\eta-\mu$ and $\alpha-\mu$ multipath fading conditions. 
TABLE I

MGF OF SNR AND ITS $n$ TH ORDER DERIVATIVE FOR THE GENERALIZED AND NON-LINEAR FADING DistRIBUtIONS

\begin{tabular}{|c|c|c|}
\hline Fading Channel & MGF of SNR, $M_{\gamma}(s)$ & $n$th order derivative of the MGF, $M_{\gamma}^{(n)}(s)$ \\
\hline$\kappa-\mu$ & $\left(\frac{(1+\kappa) \mu}{(1+\kappa) \mu+\bar{\gamma} s}\right) \exp \left(-\frac{\kappa \mu \bar{\gamma} s}{2 m \bar{\gamma} s}\right)$ & $M_{\gamma}(s) \sum_{i=0}^{n}\left(\begin{array}{c}n \\
i\end{array}\right)(\mu)_{n-i}\left(-\frac{\bar{\gamma}}{(1+\kappa) \mu+\bar{\gamma} s}\right)^{n-i} B_{i}$ \\
\hline $\begin{array}{l}\kappa-\mu \text { Extreme } \\
\eta-\mu\end{array}$ & $\left(\frac{e^{-\frac{2 m \hat{\gamma} s}{2 m+\bar{\gamma} s}}-e^{-2 m}}{(2(h-H) \mu+\bar{\gamma} s)(2(h+H) \mu+\bar{\gamma} s)}\right)^{\mu}$ & $\begin{array}{c}e^{-\frac{2 m \hat{\gamma} s}{2 m+\hat{\gamma} s}} B_{n} \\
\sum^{n}\left(\begin{array}{c}n \\
i\end{array}\right) \frac{(-1)^{n} \bar{\gamma}^{n}(\mu)_{i}(\mu)_{n-i}}{\left.(2(h-H) \mu+\bar{\gamma} s)^{\mu+i}(2 h+H) \mu+\bar{\gamma} s\right)^{\mu+n-i}}\end{array}$ \\
\hline$\alpha-\mu$ & $\begin{array}{l}\frac{\alpha \mu_{k} \frac{1}{2} l \frac{\alpha \mu-1}{2}}{2 \Gamma(\mu) \bar{\gamma} \frac{\alpha \mu}{2}(2 \pi) \frac{l+k-2}{2}{ }_{s} \frac{\alpha \mu}{2}} \\
\times G_{l, k}^{k, l}\left(\left(\frac{\mu}{\bar{\gamma}^{\frac{\alpha}{2}}}\right)^{k}{\frac{l}{k^{k} s^{l}}}^{l} \mid \Delta\left(\begin{array}{c}\Delta\left(k, 1-\frac{\alpha \mu}{2}\right) \\
\Delta(k, 0)\end{array}\right.\right.\end{array}$ & $\begin{array}{l}\frac{\alpha \mu \mu_{k} \frac{1}{2} l \frac{\alpha \mu-1}{2}}{2 \Gamma(\mu) \bar{\gamma} \frac{\alpha \mu}{2}(2 \pi) \frac{l+k-2}{2}} \sum_{i=0}^{n}\left(\begin{array}{l}n \\
i\end{array}\right) \frac{(-1)^{n-i} l^{i}\left(\frac{\alpha \mu}{2}\right)_{n-i}}{s^{\frac{\alpha \mu}{2}+n}} \\
\times G_{2 l, k+l}^{k+l, l}\left(\left(\frac{\mu}{\bar{\gamma}^{\frac{\alpha}{2}}}\right)^{k} \frac{l^{l}}{k^{k} s^{l} l} \mid \begin{array}{c}\Delta\left(l, 1-\frac{\alpha \mu}{2}\right), \Delta(l, 1-i) \\
\Delta(k, 0), \Delta(l, 1)\end{array}\right.\end{array}$ \\
\hline
\end{tabular}

\section{A. Average AUC Over $\kappa-\mu$ Fading Channels}

By substituting (7) in (66), we are required to evaluate analytically the following integral

$$
\mathcal{I}_{8}=\int_{\gamma_{T}}^{\infty} \gamma^{\frac{\mu-1}{2}+i} e^{-\frac{1+2 \zeta}{2} \gamma} I_{\mu-1}(2 \sqrt{\kappa \mu \zeta \gamma}) \mathrm{d} \gamma .
$$

By letting $t^{2}=(1+2 \zeta) \gamma$ and after some algebraic manipulations, the $\mathcal{I}_{8}$ integral can be expressed as

$\mathcal{I}_{8}=\frac{2 e^{\frac{2 \kappa \mu \zeta}{1+2 \zeta}}}{(1+2 \zeta)^{\frac{\mu+1}{2}+i}} Q_{\mu+2 i, \mu-1}\left(2 \sqrt{\frac{\kappa \mu \zeta}{1+2 \zeta}}, \sqrt{(1+2 \zeta) \gamma_{T}}\right)$

where $Q_{m, n}(a, b)$ denotes the Nuttall $Q$-function [48]. Based on this, it is evident that by substituting (68) into (66) yields

$$
\begin{aligned}
& \overline{\mathrm{AUC}}_{\kappa-\mu}^{\mathrm{SSC}}=1- F_{\gamma}\left(\gamma_{T}\right) \overline{\mathrm{CAUC}}-\sum_{l=0}^{u-1} \sum_{i=0}^{l}\left(\begin{array}{c}
l+u-1 \\
l-i
\end{array}\right) \\
& \times \frac{\zeta^{\frac{1+\mu}{2}} Q_{\mu+2 i, \mu-1}\left(\sqrt{\frac{4 \kappa \mu \zeta}{1+2 \zeta}}, \sqrt{(1+2 \zeta) \gamma_{T}}\right)}{i ! e^{\frac{\kappa \mu}{1+2 \zeta}}(\kappa \mu)^{\frac{\mu-1}{2}} 2^{l+i+u-1}(1+2 \zeta)^{\frac{\mu+1}{2}+i}}
\end{aligned}
$$

where $\overline{\mathrm{CAUC}}$ is readily obtained by Theorem 2 .

\section{B. Average AUC Over $\kappa-\mu$ Extreme Fading Channels}

By substituting (12) into (66), it immediately follows that

$$
\begin{gathered}
\overline{\mathrm{AUC}}=1-F_{\gamma}\left(\gamma_{T}\right) \overline{\mathrm{CAUC}}-\frac{2 m}{\sqrt{\bar{\gamma}} e^{2 m}} \sum_{l=0}^{u-1} \sum_{i=0}^{l}\left(\begin{array}{c}
l+u-1 \\
l-i
\end{array}\right) \\
\times\left(\frac{1}{2}\right)^{l+i+u} \frac{1}{i !} \underbrace{\int_{\gamma_{T}}^{\infty} \gamma^{i-\frac{1}{2}} e^{-\left(\frac{1}{2}+\frac{2 m}{\bar{\gamma}}\right) \gamma} I_{1}\left(4 m \sqrt{\frac{\gamma}{\bar{\gamma}}}\right) \mathrm{d} \gamma}_{\mathcal{I}_{9}} \\
-\frac{e^{-2 m}}{2^{u+1} \sqrt{\bar{\gamma}}} \sum_{l=0}^{u-1} \sum_{i=0}^{l}\left(\begin{array}{c}
l+u-1 \\
l-i
\end{array}\right) \frac{1}{i ! 2^{l+i}} \underbrace{\int_{\gamma_{T}}^{\infty} \gamma^{i-\frac{1}{2}} e^{-\frac{\gamma}{2}} \delta(\gamma) \mathrm{d} \gamma}_{\mathcal{I}_{10}} .
\end{gathered}
$$

By letting $t^{2}=(1+4 m / \bar{\gamma}) \gamma$ and after some algebraic manipulations, it becomes evident that $\mathcal{I}_{9}$ can be expressed in closed-form in terms of the Nuttall $Q$-function yielding

$$
\mathcal{I}_{9}=\frac{4 m e^{-2 m} \bar{\gamma}^{i} e^{\frac{8 m^{2}}{\gamma}+4 m}}{(\bar{\gamma}+4 m)^{i+\frac{1}{2}}} Q_{2 i, 1}\left(\frac{4 m}{\sqrt{\bar{\gamma}+4 m}}, \sqrt{\frac{\gamma_{T}(\bar{\gamma}+4 m)}{\bar{\gamma}}}\right) .
$$

Thus, substituting (71) into (70) and given that $\mathcal{I}_{10}=0$ yields

$$
\begin{array}{r}
\overline{\mathrm{AUC}}_{\kappa-\mu \text { Ext. }}^{\mathrm{SSC}}=1-F_{\gamma}\left(\gamma_{T}\right) \overline{\mathrm{CAUC}}-\sum_{l=0}^{u-1} \sum_{i=0}^{l}\left(\begin{array}{c}
l+u-1 \\
l-i
\end{array}\right) \\
\times \frac{m \bar{\gamma}^{i} Q_{2 i, 1}\left(\frac{4 m}{\sqrt{\bar{\gamma}+4 m}}, \sqrt{\frac{\gamma_{T}(\bar{\gamma}+4 m)}{\bar{\gamma}}}\right)}{i ! e^{\frac{2 m}{\bar{\gamma}+4 m}}(\bar{\gamma}+4 m)^{i+\frac{1}{2}} 2^{l+i+u-2}} .
\end{array}
$$

\section{Average AUC for SSC Over $\eta-\mu$ Fading Channels}

By substituting (14) in (66), it immediately follows that

$$
\begin{aligned}
\overline{\mathrm{AUC}}_{\eta-\mu}^{\mathrm{SSC}} & =\left[1+F_{\gamma}\left(\gamma_{T}\right)\right] \overline{\mathrm{AUC}}-F_{\gamma}\left(\gamma_{T}\right) \\
& +\frac{2 \sqrt{\pi} \mu^{\mu+\frac{1}{2}} h^{\mu}}{\Gamma(\mu) H^{\mu-\frac{1}{2}} \bar{\gamma}^{\mu+\frac{1}{2}}} \sum_{l=0}^{u-1} \sum_{i=0}^{l}\left(\begin{array}{c}
l+u-1 \\
l-i
\end{array}\right)\left(\frac{1}{2}\right)^{l+i+u} \\
& \times \frac{1}{i !} \underbrace{\int_{0}^{\gamma_{T}} \gamma^{i+\mu-\frac{1}{2}} e^{-\left(\frac{2 \mu h}{\bar{\gamma}}+\frac{1}{2}\right) \gamma} I_{\mu-\frac{1}{2}}\left(\frac{2 \mu H}{\bar{\gamma}} \gamma\right) \mathrm{d} \gamma}_{\mathcal{I}_{11}}
\end{aligned}
$$

Notably, the above integral can be expressed in terms of the incomplete Lipschitz-Hankel function [57], [60]. Therefore, by setting $\bar{\gamma} y=2 \mu H \gamma$ it follows that

$$
\mathcal{I}_{11}=\int_{0}^{\gamma_{T}} \gamma^{i+\mu-\frac{1}{2}} e^{-\left(\frac{\bar{\gamma}+4 \mu h}{2 \gamma}\right) \gamma} I_{\mu-\frac{1}{2}}\left(\frac{2 \mu H}{\bar{\gamma}} \gamma\right) \mathrm{d} \gamma .
$$

To this effect, by substituting (74) into (73) and after some algebraic manipulations, one obtains

$$
\begin{aligned}
\overline{\mathrm{AUC}_{\eta-\mu}^{\mathrm{SSC}}=} & {\left[1+F_{\gamma}\left(\gamma_{T}\right)\right] \overline{\mathrm{AUC}}-F_{\gamma}\left(\gamma_{T}\right)+\sum_{l=0}^{u-1} \sum_{i=0}^{l} \sqrt{\pi} h^{\mu} \bar{\gamma}^{i} } \\
& \times\left(\begin{array}{c}
l+u-1 \\
l-i
\end{array}\right) \frac{I e_{i+\mu-\frac{1}{2}, \mu-\frac{1}{2}}\left(\frac{2 \mu H \gamma_{T}}{\bar{\gamma}} ; \frac{4 \mu h+\bar{\gamma}}{4 \mu H}\right)}{2^{l+2 i+u+\mu-\frac{1}{2}} \mu^{i} H^{2 \mu+i} i ! \Gamma(\mu)}
\end{aligned}
$$


where $I e_{m, n}(x ; z)$ and $\gamma(a, x)$ denote the modified Bessel function of the first kind based incomplete Lipschitz-Hankel integrals and the lower incomplete gamma function, respectively. Importantly, with the aid of [60, eq. (53)], equation (75) can be expressed according to (76), as shown bottom of this page, for the specific, yet useful, case that $\mu \in \mathbb{N}$.

\section{Average AUC for SSC Over $\alpha-\mu$ Fading Channels}

An analytic expression is also derived for the corresponding $\overline{\mathrm{AUC}}$ over $\alpha-\mu$ fading channels. To this end, by substituting (21) in (66) and expanding $\exp \left(-\mu \gamma^{\frac{\alpha}{2}} \bar{\gamma}^{-\frac{\alpha}{2}}\right)$ yields

$$
\mathcal{I}_{12}=\sum_{j=0}^{\infty} \frac{(-1)^{j}}{j !} \mu^{j} \bar{\gamma}^{-\frac{\alpha j}{2}} \int_{0}^{\gamma_{T}} \gamma^{i+\frac{\alpha j}{2}+\frac{\alpha \mu}{2}-1} e^{-\frac{\gamma}{2}} \mathrm{~d} \gamma
$$

Based on this and by utilizing [48, eq. (3.381.3)], one obtains

$$
\begin{aligned}
\overline{\mathrm{AUC}}_{\alpha-\mu}^{\mathrm{SSC}}=\overline{\mathrm{AUC}} & -F_{\gamma}\left(\gamma_{T}\right) \overline{\mathrm{CAUC}}+\sum_{l=0}^{u-1} \sum_{i=0}^{l} \sum_{j=0}^{\infty}\left(\begin{array}{c}
l+u-1 \\
l-i
\end{array}\right) \\
& \times \frac{(-1)^{j} \alpha \mu^{j+\mu} \gamma\left(i+\frac{\alpha j}{2}+\frac{\alpha \mu}{2}, \frac{\gamma_{T}}{2}\right)}{2^{l-\frac{\alpha(j+\mu)}{2}+u+1} \bar{\gamma}^{\frac{\alpha(j+\mu)}{2}} \Gamma(\mu) i ! j !} .
\end{aligned}
$$

It is noted that the $\overline{\mathrm{AUC}}$ and $\overline{\mathrm{CAUC}}$ terms can be determined with the aid of Theorem 4 and Theorem 5, respectively. Furthermore, the series in (78) is convergent and can be truncated accurately after only few terms.

It is finally recalled that the performance gains by the use of SSC are largely based on the effective determination of $\gamma_{T}$. Thus, in order to maximize the $\overline{\mathrm{AUC}}_{\mathrm{SSC}}$, the $\gamma_{T}$ threshold must be determined accurately. To this end, by evaluating

$$
\frac{\partial \overline{\mathrm{AUC}}_{\mathrm{SSC}}}{\partial \gamma_{T}}=0
$$

and after some algebraic manipulations, the desired optimal value of threshold $\gamma_{T}^{*}$ is given by

$$
\frac{\partial \overline{\mathrm{AUC}}^{\mathrm{SSC}}}{\partial \gamma_{T}}=\overline{\mathrm{AUC}} \frac{\partial F_{\gamma}\left(\gamma_{T}\right)}{\partial \gamma_{T}}-A\left(\gamma_{T}\right) f_{\gamma}\left(\gamma_{T}\right)=0
$$

while $\overline{\mathrm{AUC}}=A\left(\gamma_{T}^{*}\right)$. Therefore, the optimal value of the SSC SNR threshold for each case renders the value of AUC under AWGN equal to the value of the corresponding average AUC.

To the best of the authors knowledge, the derived analytic expressions in the present contribution have not been previously reported in the open technical literature.

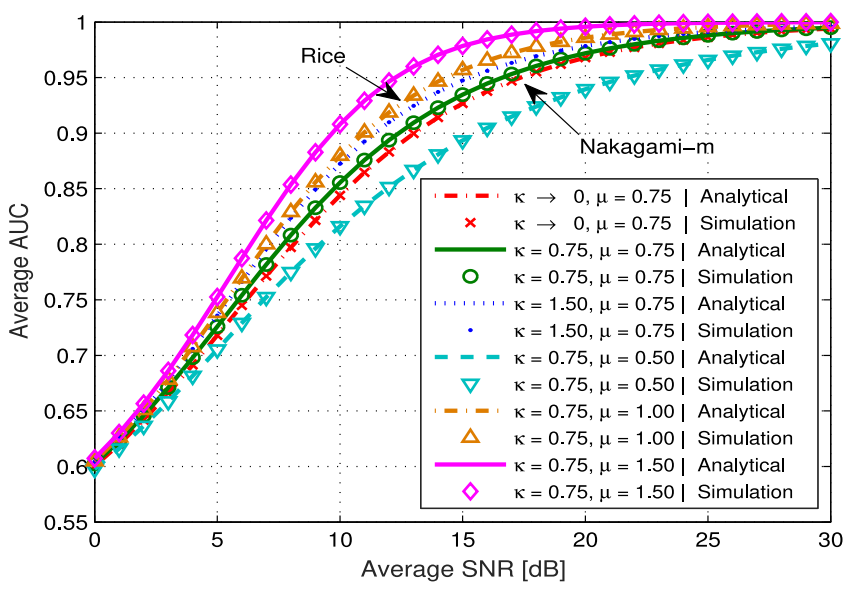

Fig. 1. Average AUC versus average SNR for $\kappa-\mu$ fading conditions with $u=5$, and different $\kappa$ and $\mu$ values.

\section{NUMERICAL RESULTS AND DISCUSSION}

The aim of this Section is threefold: i) to validate the accuracy of the derived analytical frameworks in Sections III-V; ii) to analyze the performance of the energy detection under the effects of multipath and fading non-linearity; iii) to quantify and compare the performance gains by SLC and SSC diversity schemes and determine the compensation of the detrimental effects of fading.

Fig. 1 illustrates the $\overline{\mathrm{AUC}}$ over $\kappa-\mu$ fading channels for $u=5$ and different values of $\kappa$ and $\mu$. As expected, the energy detector exhibits better detection capability for larger values of $\kappa$ and $\mu$, which is mainly achieved thanks to the increased power reception by the existing dominant components. Indicatively, for the case of $\bar{\gamma}=10 \mathrm{~dB}$ and $\mu=0.75$, the corresponding $\overline{\mathrm{AUC}}$ for the case of $\kappa=1.5$ is $2 \%$ higher than that for $\kappa=0.75$. In the same context, when $\kappa=0.75$, the $\overline{\mathrm{AUC}}$ for $\mu=1$ is nearly $7.8 \%$ higher than for the case of $\mu=0.5$. The flexibility of the $\kappa-\mu$ distribution is also demonstrated as the $\overline{\text { AUC }}$ for $\kappa \rightarrow 0$ and $\mu=0.75$ coincides with that for the case of Nakagami $-m$ fading for $m=0.75$. Likewise, the special case that $\kappa=0.75$ and $\mu=1$ coincides with the Ricean case for Rice parameter with $K=0.75$, or equivalently with that of Nakagami $-n$ with $n^{2}=0.75$. By recalling that $\kappa$ and $K$ parameters, in $\kappa-\mu$ and Rice distributions respectively, denote the ratio between the total power of the dominant components and the total power of the scattered waves, Fig. 1 also shows the reduced performance when a dominant component does not exist (i.e. $\kappa=0.75$ ) along

$$
\begin{aligned}
\overline{\mathrm{AUC}}_{\eta-\mu}^{\mathrm{SSC}} & =\left[1+F_{\gamma}\left(\gamma_{T}\right)\right] \overline{\mathrm{AUC}}-F_{\gamma}\left(\gamma_{T}\right) \\
& +\frac{\mu^{\mu} h^{\mu}}{2^{u-\mu} \Gamma(\mu) H^{\mu}} \sum_{l=0}^{u-1} \sum_{i=0}^{l} \sum_{j=0}^{\mu-1}\left(\begin{array}{c}
l+u-1 \\
l-i
\end{array}\right) \frac{\bar{\gamma}^{i} \Gamma(\mu+j) \gamma\left(i-j+\mu,(4 \mu(h-H)+\bar{\gamma}) \frac{\gamma_{T}}{2 \bar{\gamma}}\right)}{2^{l+3 j} i ! j ! \Gamma(\mu-j)(-\mu H)^{j}(4 \mu(h-H)+\bar{\gamma})^{i-j+\mu}} \\
& +\frac{(-1)^{\mu} \mu^{\mu} h^{\mu}}{2^{u-\mu} \Gamma(\mu) H^{\mu}} \sum_{l=0}^{u-1} \sum_{i=0}^{l} \sum_{j=0}^{\mu-1}\left(\begin{array}{c}
l+u-1 \\
l-i
\end{array}\right) \frac{\bar{\gamma}^{i} \Gamma(\mu+j) \gamma\left(i-j+\mu,(4 \mu(h+H)+\bar{\gamma}) \frac{\gamma_{T}}{2 \bar{\gamma}}\right)}{2^{l+3 j} i ! j ! \Gamma(\mu-j)(\mu H)^{j}(4 \mu(h+H)+\bar{\gamma})^{i-j+\mu}}
\end{aligned}
$$




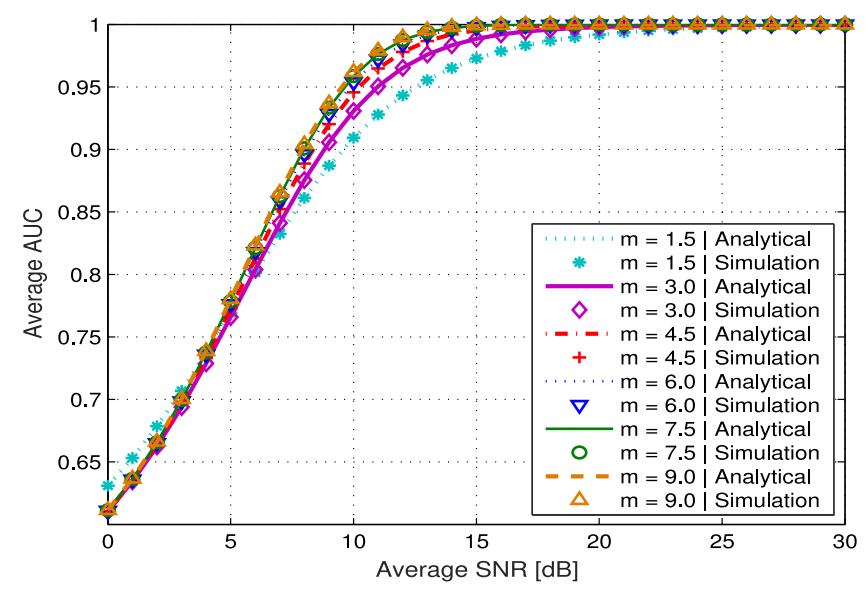

Fig. 2. Average AUC versus average SNR for $\kappa-\mu$ extreme fading conditions with $u=5$, and different values of $m$.

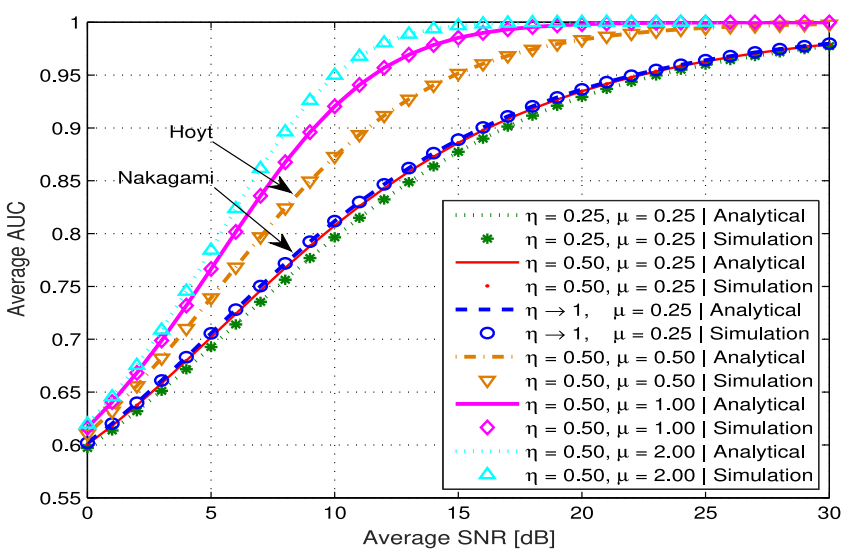

Fig. 3. Average AUC versus average SNR for $\eta-\mu$ fading conditions with $u=4$, and different values of $\eta$ and $\mu$.

with the poor characterization of Rice distribution, which unlike $\kappa-\mu$ model fails to account for fading conditions that correspond to different values of $\mu$. In the same context, Fig. 2 illustrates the $\overline{\mathrm{AUC}}$ under $\kappa-\mu$ extreme fading conditions with respect to the corresponding average SNR for different values of $m$ with $u=5$. It is evident that the performance of the energy detector is highly dependent upon the severity of fading, and it improves substantially as $m$ increases. For example, for the case of $\bar{\gamma}=10 \mathrm{~dB}$, the $\overline{\mathrm{AUC}}$ for $m=3$ is nearly $2.4 \%$ higher than that for $m=1.5$.

Fig. 3 illustrates the average $\overline{\mathrm{AUC}}$ over $\eta-\mu$ fading channels for different $\eta$ and $\mu$ values under Format ${ }^{1}$ I. It is shown that the energy detector performs adequately as $\mu$ increases, with fixed $\eta$, due to the relative advantages of multipath effect. This is also the case for increasing $\eta$ thanks to the increased received power of the in-phase components. Yet, it is noted that since the $\eta-\mu$ distribution is symmetrical around $\eta=1$, within $0<$ $\eta^{-1} \leq 1$ the energy detector exhibits better performance for lower values of $\eta$. For example, for the case of $\bar{\gamma}=10 \mathrm{~dB}$ and $\mu=0.25$, the $\overline{\mathrm{AUC}}$ for $\eta=0.5$ is nearly $1.4 \%$ higher than for

\footnotetext{
${ }^{1}$ Respective results for the useful scenario of Format II of the $\eta-\mu$ fading model can be readily obtained by a simple change of variables.
}

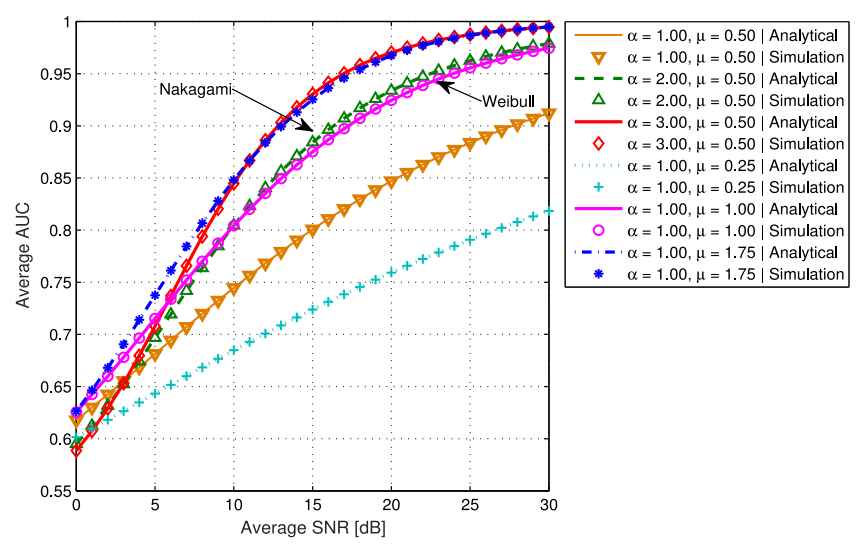

Fig. 4. Average AUC versus average SNR for $\alpha-\mu$ fading conditions with $u=5$, and different values of $\alpha$ and $\mu$.

$\eta=0.25$. Likewise, when $\eta=0.5$, the $\overline{\mathrm{AUC}}$ is $5.5 \%$ higher for $\mu=1$ than for $\mu=0.5$. Regarding relation to multipath effects characterized by more conventional fading models, the special case for $\eta \rightarrow 1$ and $\mu=0.25$ coincides with the case of Nakagami $-m$ fading for $m=0.5$, which represents a worst case scenario. Moreover, the special case of $\mu=0.5$ and $\eta=0.5$ coincides with that for Hoyt fading conditions, with $b=1 / 3$, or equivalently with that of Nakagami- $-q$ model with $q^{2}=0.5$.

Likewise, Fig. 4 illustrates the $\overline{\text { AUC }}$ over $\alpha-\mu$ fading channels for $u=5$ and different values of $\alpha$ and $\mu$. One can observe that the energy detector performs, as expected, better as $\alpha$ and $\mu$ increase due to advantages of the non-linearity of the medium and the number of multipath clusters, respectively. For example, for the case of $\bar{\gamma}=10 \mathrm{~dB}$ and $\mu=0.5$, the $\overline{\mathrm{AUC}}$ for $\alpha=2$ is nearly $8 \%$ higher than for $\alpha=1$. In the same context, when $\alpha=1$, the $\overline{\mathrm{AUC}}$ is $17 \%$ higher for $\mu=1$ than for $\mu=0.25$. It is also shown that the case for $\alpha=2$ and $\mu=0.5$ coincides with the most severe Nakagami $-m$ fading conditions i.e. $m=0.5$. Likewise, the case for $\mu=1$ and $\eta=1$ coincides with Weibull fading conditions with severity $\beta=1$. Overall, it is seen that the effect of the fading non-linearity is more significant than that of $\mu$. In fact, this also holds when compared to the parameters $\kappa, m$ and $\eta$ of the $\kappa-\mu, \kappa-\mu$ extreme and $\eta-\mu$ distributions [54]. Thus, it is evident that neglecting the effects of fading non-linearity will lead to inaccurate results regarding the performance of energy detectors over multipath fading channels. It is also noted that the exact analytic results in the above figures are in excellent agreement with the numerical results from computer simulations, which verifies the correctness of the derived analytic expressions.

Figs. 5-8 illustrate the performance of ED over $\kappa-\mu, \kappa-\mu$ extreme, $\eta-\mu$ and $\alpha-\mu$ fading channels, respectively, for the case of SLC. It is shown that the detrimental effects of fading are practically compensated as the ED performance is significantly improved in severe fading conditions. Specifically, Fig. 5 demonstrates the $\overline{\text { AUC }}$ vs $\bar{\gamma}$ for the case of $\kappa-\mu$ fading with $\kappa=3, \mu=1.8$ and $u=4$. It is shown that increasing the number of diversity branches provides a significant compensation for fading degradation. This is also the case in $\kappa-\mu$ extreme, $\eta-\mu$ and $\alpha-\mu$ fading conditions, as illustrated by the $\overline{\mathrm{AUC}}$ vs $\bar{\gamma}$ curves for $m=1.8, u=4 ; \eta=0.5, \mu=0.7, u=3$; 


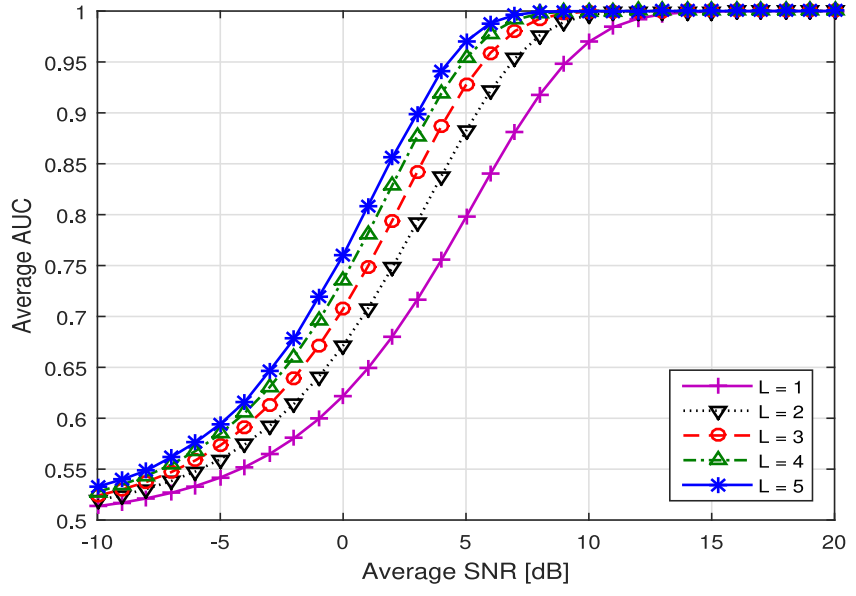

Fig. 5. Average AUC versus average SNR for $L$-branch SLC diversity receptions over $\kappa-\mu$ fading channels ( $u=4, \kappa=4$ and $\mu=1.5$ ).

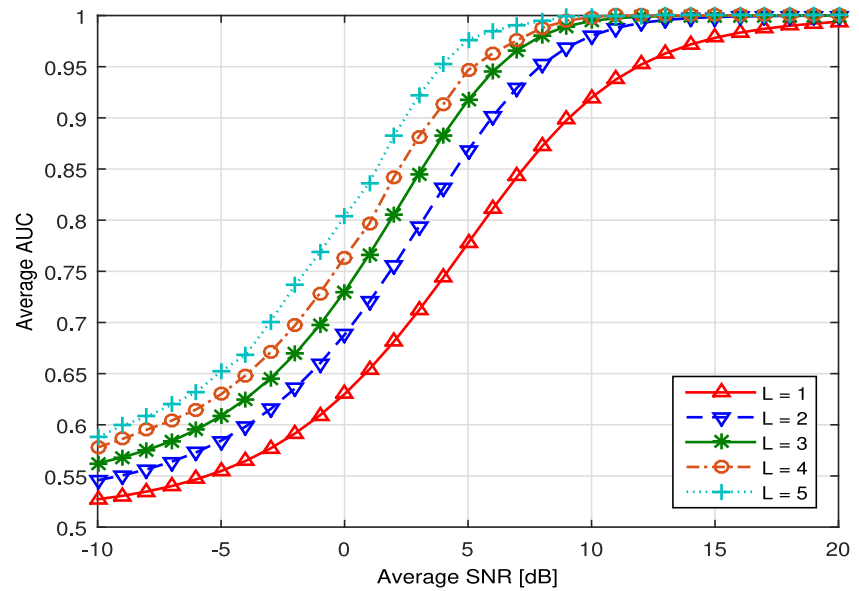

Fig. 6. Average AUC versus average SNR for $L$-branch SLC diversity receptions over $\kappa-\mu$ extreme fading channels $(u=4, m=1.8)$.

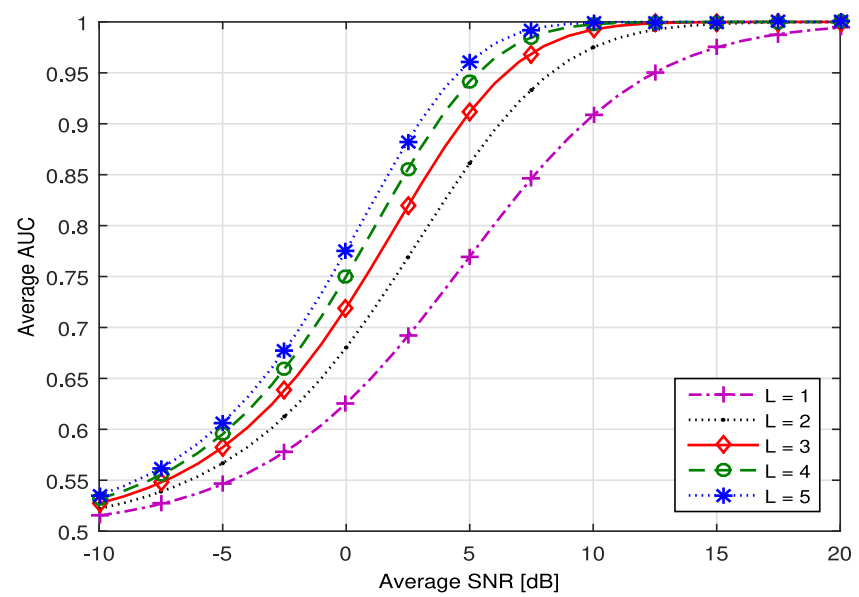

Fig. 7. Average AUC versus average SNR for $L$-branch SLC diversity receptions over $\eta-\mu$ fading channels ( $u=3, \eta=0.5$ and $\mu=0.7$ ).

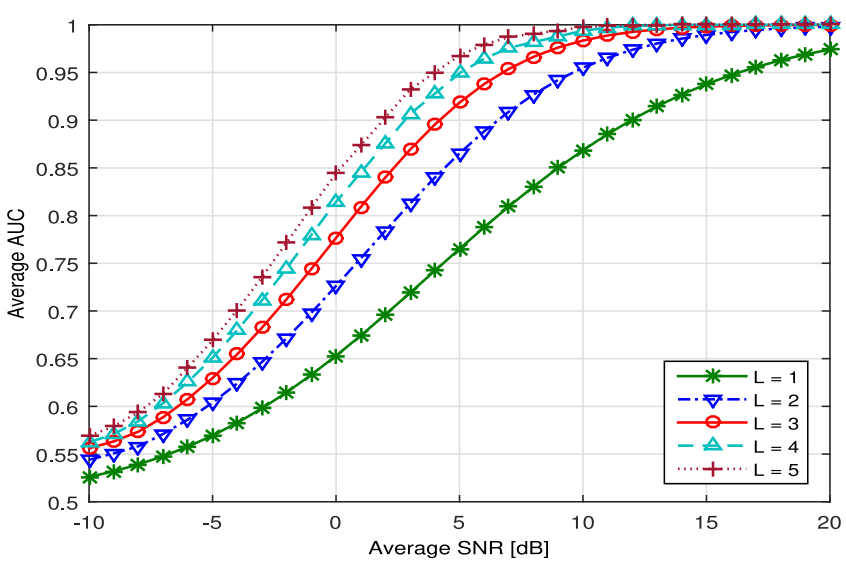

Fig. 8. Average AUC versus average SNR for $L$-branch SLC diversity receptions over $\alpha-\mu$ fading channels ( $u=3, \alpha=0.8$ and $\mu=2.5$ ).

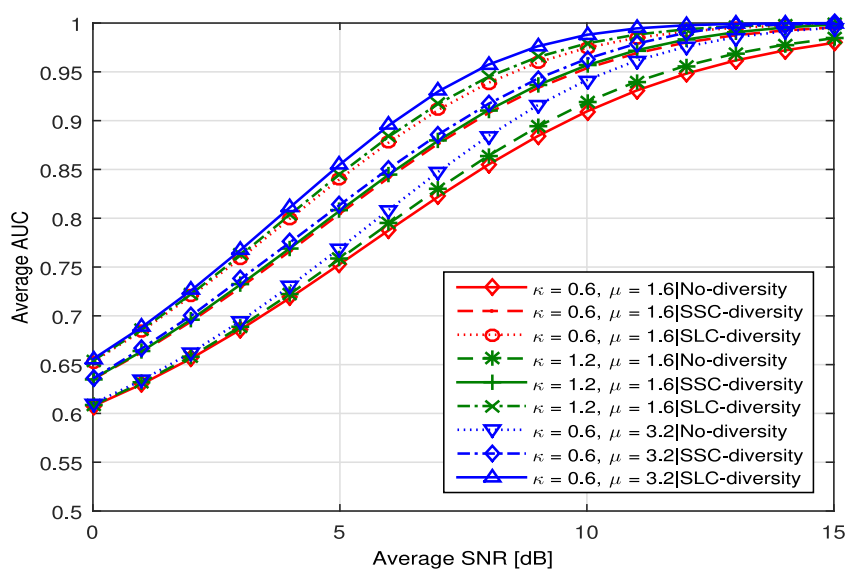

Fig. 9. Average AUC versus average SNR for dual branch of SSC and SLC diversity schemes over $\kappa-\mu$ fading channels with $u=5$.

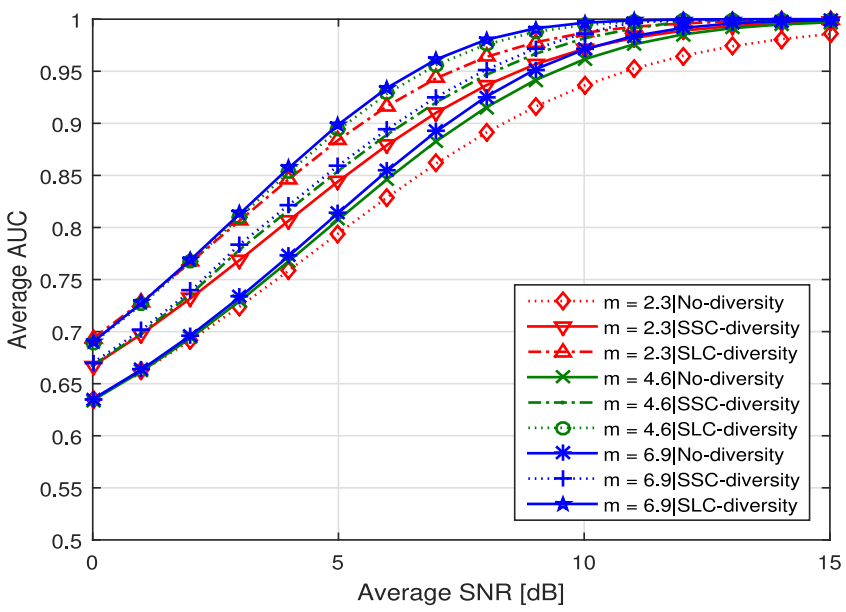

Fig. 10. Average AUC versus average SNR for dual branch of SSC and SLC diversity schemes over $\kappa-\mu$ extreme fading channels with $u=3$. 


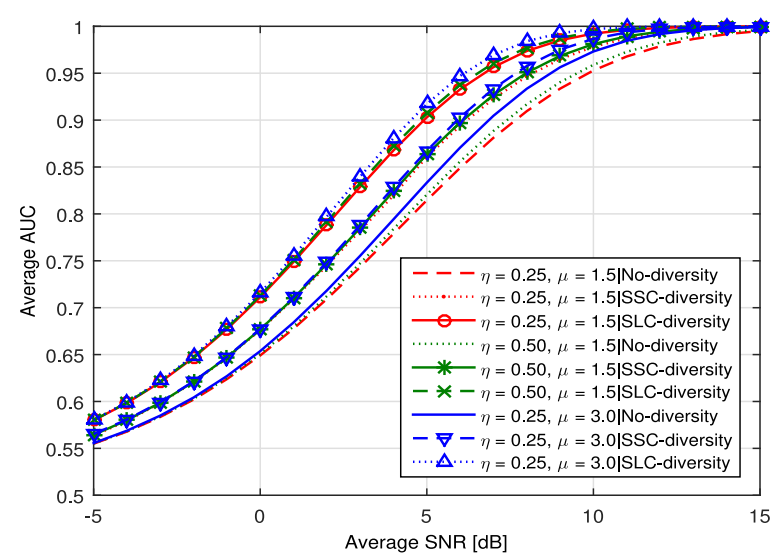

Fig. 11. Average AUC versus average SNR for dual branch of SSC and SLC diversity schemes over $\eta-\mu$ fading channels with $u=2$.

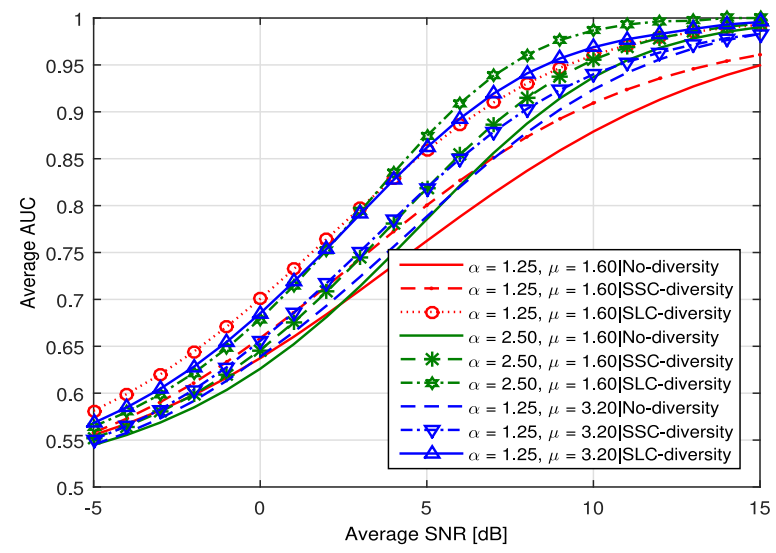

Fig. 12. Average AUC versus average SNR for dual branch of SSC and SLC diversity schemes over $\alpha-\mu$ fading channels with $u=3$.

and $\alpha=0.8, \mu=2.5, u=3$, respectively. To this effect, it is generally noted that the aforementioned performance gains are particularly evident in moderate and high SNR regimes where $3-5$ diversity branches are practically sufficient for ensuring adequate and robust detection performance for any type and severity of multipath fading conditions.

Further results on the ED performance over $\kappa-\mu, \kappa-\mu$ extreme, $\eta-\mu$ and $\alpha-\mu$ fading channels with diversity reception are depicted in Figs. 9-12, respectively. This is performed by means of $\overline{\mathrm{AUC}}$ vs $\bar{\gamma}$ curves for dual SSC $(L=2)$ and different fading parameters. Notably, the corresponding results for the case of SLC are also depicted for comparison, while the optimum switching thresholds that maximize the average AUC in SSC have been used for each value of $\bar{\gamma}$. The $\overline{\mathrm{AUC}}$ curves depict the significantly improved performance achieved also when using SSC. This demonstrates the overall diversity advantages on the energy detector capability compared to the conventional single-channel communication scenario. For example, in $\kappa-\mu$ fading conditions for $\bar{\gamma}=5 \mathrm{~dB}$ and $u=5$ with $\kappa=0.6$ and $\mu=1.6$, the $\overline{\text { AUC }}$ over the single branch operation increased for about $7.3 \%$ and $11.6 \%$ for SSC and SLC, respectively. In the case of $\kappa-\mu$ extreme fading for $\bar{\gamma}=8 \mathrm{~dB}$ and $u=3$ with $m=2.3$, the $\overline{\mathrm{AUC}}$ outperforms the single branch operation by $5.1 \%$ and $7.3 \%$ for SSC and SLC, respectively. Likewise, in the case of $\eta-\mu$ fading for $\bar{\gamma}=7 \mathrm{~dB}$ and $u=2$ with $\eta=0.5$ and $\mu=1.5$, the $\overline{\mathrm{AUC}}$ outperforms the single branch operation by $4.3 \%$ and $8.1 \%$ for SSC and SLC, respectively. Finally, in the $\alpha-\mu$ case for $\bar{\gamma}=10 \mathrm{~dB}$ and $u=3$ with $\alpha=1.25$ and $\mu=3.2$, the value of $\overline{\mathrm{AUC}}$ outperforms the single branch operation for about $1.8 \%$ and $4.9 \%$ for SSC and SLC, respectively.

\section{CONCLUSION}

A comprehensive analytical framework was derived for the average AUC in energy detection based spectrum sensing over $\kappa-\mu, \kappa-\mu$ extreme, $\eta-\mu$ and $\alpha-\mu$ fading channels for both single-channel and multi-channel communication scenarios. This was realized by capitalizing on the semi-analytic MGF approach which led to the derivation of elegant and tractable analytic expressions. These expressions are given in closed-form and their asymptotic behavior at high and low SNR regimes was thoroughly analyzed for providing insights on the effect of the involved parameters on the detection performance. The offered results exhibit that energy detection is highly affected by multipath fading conditions and particularly in the detrimental case of non-linear fading effects. Yet, it was shown that square-law-combining and switch-and-stay combining methods can improve significantly the detection performance and are capable of providing compensation for the detrimental effects of severe fading conditions. More importantly, it was shown that three to five diversity branches are practically sufficient to ensure acceptable and robust performance in all types and severity of multipath fading effects.

\section{APPENDIX I \\ PROOF OF THEOREM 1}

Given that

$$
M_{\gamma}(s)=\int_{0}^{\infty} e^{s \gamma} f(\gamma) \mathrm{d} \gamma
$$

the MGF of $\kappa-\mu$ extreme distribution is expressed as

$$
\begin{aligned}
M_{\gamma}(s)= & \frac{2 m e^{-2 m}}{\sqrt{\bar{\gamma}}} \underbrace{\int_{0}^{\infty} \gamma^{-\frac{1}{2}} e^{-\left(\frac{2 m}{\bar{\gamma}}+s\right) \gamma} I_{1}\left(4 m \sqrt{\frac{\gamma}{\bar{\gamma}}}\right) \mathrm{d} \gamma}_{\mathcal{I}_{1}} \\
& +\frac{e^{-2 m}}{2 \sqrt{\bar{\gamma}}} \underbrace{\int_{0}^{\infty} \gamma^{-\frac{1}{2}} e^{-s \gamma} \delta(\gamma) \mathrm{d} \gamma}_{\mathcal{I}_{2}} .
\end{aligned}
$$

With the aid of [48, eq. (6.643.2)] and [48, eq. (9.220.2)], the $\mathcal{I}_{1}$, integral can be expressed as

$$
\mathcal{I}_{1}=\frac{2 m \sqrt{\bar{\gamma}}}{2 m+\bar{\gamma} s}{ }_{1} F_{1}\left(1 ; 2 ; \frac{4 m^{2}}{2 m+\bar{\gamma} s}\right)
$$

where ${ }_{1} F_{1}(\cdot)$ denotes the Kummer's hypergeometric function. To this effect and by using [61, eq. (07.20.03.0052.01)] and considering $\mathcal{I}_{2}=0$ yields (13), which completes the proof. 


\section{APPENDIX II}

PROOF OF THEOREM 2

By substituting (7) in (27) it follows that

$$
\begin{aligned}
\overline{\mathrm{AUC}}_{\kappa-\mu}=1- & \frac{e^{-\kappa \mu} \zeta^{\frac{\mu+1}{2}}}{2^{u}(\kappa \mu)^{\frac{\mu-1}{2}}} \sum_{l=0}^{u-1} \sum_{i=0}^{l}\left(\begin{array}{c}
l+u-1 \\
l-i
\end{array}\right) \frac{1}{2^{l+i} i !} \\
& \times \underbrace{\int_{0}^{\infty} \frac{\gamma^{i+\frac{\mu-1}{2}}}{e^{\left(\frac{1}{2}+\zeta\right) \gamma}} I_{\mu-1}(2 \sqrt{\kappa \mu \zeta \gamma}) \mathrm{d} \gamma}_{\mathcal{I}_{3}} .
\end{aligned}
$$

The $\mathcal{I}_{3}$ integral can be expressed in closed-form with the aid of [48, eq. (6.643.2)]. Based on this and applying the identity

$$
{ }_{1} F_{1}(a ; b ; z)=\exp (z){ }_{1} F_{1}(b-a ; b ;-z)
$$

in $[48$, eq. (9.212.1)] one obtains straightforwardly

$$
\mathcal{I}_{3}=\frac{(\mu)_{i}(\kappa \mu \zeta)^{\frac{\mu-1}{2}}}{e^{-\frac{2 \kappa \mu \zeta}{1+2 \zeta}}}\left(\frac{2}{1+2 \zeta}\right)^{i+\mu}{ }_{1} F_{1}\left(-i ; \mu ;-\frac{2 \kappa \mu \zeta}{1+2 \zeta}\right) \text {. }
$$

By also using [48, eq. (8.972.1)] and carrying out some algebraic manipulations it follows that

$$
\begin{aligned}
\overline{\mathrm{AUC}}_{\kappa-\mu}= & 1-c_{1} \sum_{l=0}^{u-1} \sum_{i=0}^{l}\left(\begin{array}{c}
l+u-1 \\
l-i
\end{array}\right) \frac{1}{2^{l}}\left(\frac{1}{1+2 \zeta}\right)^{i} \\
& \times L_{i}^{(\mu-1)}\left(-\frac{2 \kappa \mu \zeta}{1+2 \zeta}\right)
\end{aligned}
$$

where $L_{n}^{\alpha}(x)$ denotes the Laguerre polynomial. By performing the necessary variable transformation in [48, eq. (8.970.1)] and substituting in (87) yields (29), which completes the proof.

\section{APPENDIX III \\ PROOF OF THEOREM 3}

The average AUC over $\kappa-\mu$ extreme fading channels when $u \in \mathbb{N}$ is given by (88), as shown bottom of this page. The $\mathcal{I}_{4}$ integral is identical to [48, eq. (6.643.2)] and thus, it can be expressed in terms of the Whittaker hypergeometric function, yielding

$$
\mathcal{I}_{4}=\frac{\sqrt{\bar{\gamma}} i !}{2 m}\left(\frac{2 \bar{\gamma}}{\bar{\gamma}+4 m}\right)^{i} e^{\frac{4 m^{2}}{\bar{\gamma}+4 m}} M_{-i, \frac{1}{2}}\left(\frac{8 m^{2}}{\bar{\gamma}+4 m}\right) .
$$

With the aid of [48, eq. (9.220.2)], equation (89) can be equivalently expressed as

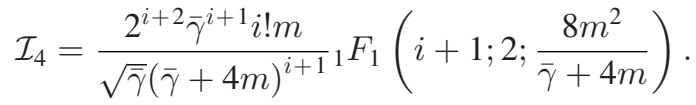

To this effect, by substituting (90) into (88) and recalling that considering $\mathcal{I}_{5}=0$, the average AUC over $\kappa-\mu$ extreme fading channels can be obtained as in (35), which completes the proof.

\section{APPENDIX IV \\ PROOF OF THEOREM 4}

By averaging (26) over the corresponding PDF in (14) it follows that

$$
\begin{aligned}
\overline{\mathrm{AUC}}_{\eta-\mu}=1- & \sum_{l=0}^{u-1} \sum_{i=0}^{l} \frac{2^{1-l-i-u} \sqrt{\pi} h^{\mu} \mu^{\mu+\frac{1}{2}}}{i ! \Gamma(\mu) H^{\mu-\frac{1}{2}} \bar{\gamma}^{\mu+\frac{1}{2}}}\left(\begin{array}{c}
l+u-1 \\
l-i
\end{array}\right) \\
& \times \underbrace{\int_{0}^{\infty} \gamma^{\mu+i-\frac{1}{2}} e^{-\frac{\bar{\gamma}+4 \mu h}{2 \bar{\gamma}} \gamma} I_{\mu-\frac{1}{2}}\left(\frac{2 \mu H}{\bar{\gamma} \gamma}\right) \mathrm{d} \gamma}_{\mathcal{I}_{6}} .
\end{aligned}
$$

The $\mathcal{I}_{6}$ integral can be expressed in closed-form using [48, eq. (7.525.1)] yielding

$$
\begin{aligned}
\mathcal{I}_{6}= & \frac{(\mu H)^{\mu-\frac{1}{2}} 2^{i+2 \mu} \bar{\gamma}^{i+\mu+\frac{1}{2}} \Gamma(i+2 \mu)}{(\bar{\gamma}+4 \mu h)^{i+2 \mu}} \\
& \times{ }_{2} \tilde{F}_{1}\left(\frac{i+2 \mu}{2}, \frac{i+2 \mu+1}{2} ; \frac{2 \mu+1}{2} ;\left(\frac{4 \mu H}{\bar{\gamma}+4 \mu h}\right)^{2}\right) .
\end{aligned}
$$

To this effect, by substituting (92) into (91) and using the binomial coefficient and the Legendre duplication formula, equation (40) is deduced, which completes the proof.

\section{APPENDIX V}

PROOF OF THEOREM 5

By substituting (21) in (27) it immediately follows that

$$
\begin{aligned}
\overline{\operatorname{AUC}}_{\alpha-\mu}=1-\sum_{i=0}^{u-1} & \sum_{j=0}^{i}\left(\begin{array}{c}
i+u-1 \\
i-j
\end{array}\right) \frac{\alpha \mu^{\mu}}{2^{i+j+u+1} \bar{\gamma}^{\frac{\alpha \mu}{2}} j ! \Gamma(\mu)} \\
& \times \underbrace{\int_{0}^{\infty} \gamma^{j+\frac{\alpha \mu}{2}-1} e^{-\frac{\gamma}{2}} e^{-\mu\left(\frac{\gamma}{\gamma}\right)^{\frac{\alpha}{2}}} \mathrm{~d} \gamma}_{\mathcal{I}_{7}} .
\end{aligned}
$$

$$
\begin{aligned}
\overline{\mathrm{AUC}}_{\kappa-\mu \text { Ext. }}= & -\frac{2 m}{e^{2 m} \sqrt{\bar{\gamma}}} \sum_{l=0}^{u-1} \sum_{i=0}^{l}\left(\begin{array}{c}
l+u-1 \\
l-i
\end{array}\right)\left(\frac{1}{2}\right)^{l+i+u} \frac{1}{i !} \underbrace{\int_{0}^{\infty} \gamma^{i-\frac{1}{2}} e^{-\left(\frac{1}{2}+\frac{2 m}{\bar{\gamma}}\right) \gamma} I_{1}\left(4 m \sqrt{\frac{\gamma}{\bar{\gamma}}}\right) \mathrm{d} \gamma}_{\mathcal{I}_{4}} \\
& -\frac{e^{-2 m}}{2 \sqrt{\bar{\gamma}}} \sum_{l=0}^{u-1} \sum_{i=0}^{l}\left(\begin{array}{c}
l+u-1 \\
l-i
\end{array}\right)\left(\frac{1}{2}\right)^{l+i+u} \frac{1}{i !} \underbrace{\int_{0}^{\infty} \gamma^{i-\frac{1}{2}} e^{-\frac{\gamma}{2}} \delta(\gamma) \mathrm{d} \gamma}_{\mathcal{I}_{5}} .
\end{aligned}
$$


Notably, the above integral can be expressed in closed-form with the aid of [52, eq. (21)] yielding

$$
\mathcal{I}_{7}=\frac{k^{\frac{1}{2}}(2 l)^{j+\frac{\alpha \mu}{2}}}{l^{\frac{1}{2}}(2 \pi)^{\frac{l+k-2}{2}}} G_{l, k}^{k, l}\left(\frac{\mu^{k} 2^{l} l^{l}}{k^{k} \bar{\gamma}^{\frac{\alpha k}{2}}} \mid \begin{array}{c}
\Delta\left(l, 1-j-\frac{\alpha \mu}{2}\right) \\
\Delta(k, 0)
\end{array}\right)
$$

which holds for both integer and non-integer values of $\alpha$ while $l$ and $k$ are positive integers that satisfy $l / k=\alpha / 2$. Thus, inserting (94) into (93) yields (48), which completes the proof.

\section{APPENDIX VI}

\section{PROOF OF LEMMA 1}

We let

$$
M_{\gamma}(s)=f(s) h(s)
$$

where

$$
f(s)=\left(\frac{(1+\kappa) \mu}{\bar{\gamma} s+(1+\kappa) \mu}\right)^{\mu}
$$

and

$$
h(s)=e^{g(s)}
$$

with

$$
g(s)=-\frac{\kappa \mu \bar{\gamma} s}{\bar{\gamma} s+(1+\kappa) \mu} .
$$

Based on this, the $n$th derivative of $f(s)$ can be expressed as follows:

$$
f^{(n)}(s)=\frac{(1+\kappa)^{\mu} \mu^{\mu}(-\bar{\gamma})^{n}(\mu)_{n}}{(\bar{\gamma} s+(1+\kappa) \mu)^{\mu+n}} .
$$

To this effect and with the aid of the Faà di Bruno's formula in (100),

$$
\begin{aligned}
& \frac{d^{n}}{d s^{n}} f(g(s)) \\
& =\sum_{k=0}^{n} f^{(k)}(g(s)) B_{n, k}\left(g^{\prime}(s), g^{\prime \prime}(s), \ldots, g^{(n-k+1)}(s)\right)
\end{aligned}
$$

and its special case for the $\exp (g(s))$ function as [57]

$$
\frac{d^{n}}{d s^{n}} e^{g(s)}=e^{g(s)} B_{n}\left(g^{\prime}(s), g^{\prime \prime}(s), \ldots, g^{(n)}(s)\right)
$$

the $n$th derivative of the MGF for (97) can be expressed as

$$
\begin{aligned}
h^{(n)}(s) & =\frac{d^{n}}{d s^{n}} e^{g(s)} \\
& =e^{g(s)} B_{n} .
\end{aligned}
$$

Hence, using the general Leibniz rule along with this and (99) yields (56), thus completing the proof.

\section{APPENDIX VII}

PROOF OF LEMMA 3

We firstly let

$$
M_{\gamma}(s)=\left(4 \mu^{2} h\right)^{\mu} f(s) g(s)
$$

where

$$
f(s)=\frac{1}{(2(h-H) \mu+\bar{\gamma} s)^{\mu}}
$$

and

$$
g(s)=\frac{1}{(2(h+H) \mu+\bar{\gamma} s)^{\mu}} .
$$

Based on this, it follows that the $n$th derivative of $f(s)$ and $g(s)$ is respectively expressed as

$$
f^{(n)}(s)=\frac{(-1)^{n} \bar{\gamma}^{n}(\mu)_{n}}{(2(h-H) \mu+\bar{\gamma} s)^{\mu+n}}
$$

and

$$
g^{(n)}(s)=\frac{(-1)^{n} \bar{\gamma}^{n}(\mu)_{n}}{(2(h+H) \mu+\bar{\gamma} s)^{\mu+n}} .
$$

As a result and with the aid of Lemma 1, equation (61) is deduced, which completes the proof.

\section{APPENDIX VIII \\ PROOF OF LEMMA 4}

By letting $f(s)=s^{-\frac{\alpha \mu}{2}}$ and

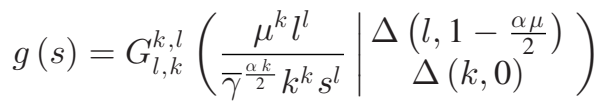

it immediately follows that

$$
M_{\gamma}(s)=\frac{\alpha \mu^{\mu} k^{\frac{1}{2}} l^{\frac{\alpha \mu-1}{2}}}{2 \Gamma(\mu) \bar{\gamma}^{\frac{\alpha \mu}{2}}(2 \pi)^{\frac{l+k-2}{2}}} f(s) g(s) .
$$

To this effect, the $n$th derivative of $f(s)$ is given by [58]

$$
f^{(n)}(s)=\frac{(-1)^{n}\left(\frac{\alpha \mu}{2}\right)_{n}}{s^{\frac{\alpha \mu}{2}+n}}
$$

whereas the $n$th derivative of $g(s)$ is determined with the aid of [58, eq. (11.b)] yielding

$$
g^{(n)}(s)=\frac{G_{2 l, k+l}^{k+l, l}\left(\frac{\mu^{k} l^{l}}{\bar{\gamma}^{\frac{\alpha k}{2}} k^{k} s^{l}} \mid \begin{array}{c}
\Delta\left(l, 1-\frac{\alpha \mu}{2}\right), \Delta(l, 1-n) \\
\Delta(l, 1), \Delta(k, 0)
\end{array}\right)}{l^{-n} s^{n}} .
$$

To this effect and using the general Leibniz rule yields (62), which completes the proof.

\section{ACKNOWLEDGMENT}

The authors would like to thank the editor and the anonymous reviewers for their constructive comments which improved the quality of the paper. This paper is dedicated to Prof. M. Renfors and to Prof. I. Andritsos on the occasion of their 65th birthday.

\section{REFERENCES}

[1] A. Bagheri, P. C. Sofotasios, T. A. Tsiftsis, A. Shahzadi, and M. Valkama, "AUC study of energy detection based spectrum sensing over $\eta-\mu$ and $\alpha-\mu$ fading channels," in Proc. IEEE Int. Conf. Commun., Jun. 2015, pp. 1410-1415.

[2] A. Bagheri, P. C. Sofotasios, T. A. Tsiftsis, A. Shahzadi, and M. Valkama "Spectrum sensing in generalized multipath fading conditions using square-law combining," in Proc. IEEE Int. Conf. Commun., Jun. 2015 pp. 7528-7533. 
[3] A. Bagheri, P. C. Sofotasios, T. A. Tsiftsis, A. Shahzadi, S. Freear, and M. Valkama, "Area under ROC curve of energy detection over generalized fading channels," in Proc. IEEE Int. Symp. Personal, Indoor Mobile Radio Commun., Aug./Sep. 2015, pp. 656-661.

[4] F. F. Digham, M.-S. Alouini, and M. K. Simon, "On the energy detection of unknown signals over fading channels," IEEE Trans. Commun., vol. 55, no. 1, pp. 21-24, Jan. 2007.

[5] C. Zhong, T. Ratnarajah, and K.-K. Wong, "Outage analysis of decodeand-forward cognitive dual-hop systems with the interference constraint in Nakagami- $m$ fading channels," IEEE Trans. Veh. Technol., vol. 60, no. 6, pp. 2875-2879, Jul. 2011.

[6] T. Q. Duong, D. B. da Costa, M. Elkashlan, and V. N. Q. Bao, "Cognitive amplify-and-forward relay networks over Nakagami- $m$ fading," IEEE Trans. Veh. Technol., vol. 61, no. 5, pp. 2368-2374, May 2012.

[7] P. J. Smith, P. A. Dmochowski, H. A. Suraweera, and M. Shafi, "The effects of limited channel knowledge on cognitive radio system capacity," IEEE Trans. Veh. Technol., vol. 62, no. 2, pp. 927-933, Feb. 2013.

[8] F. R. V Guimaraes, D. B. da Costa, T. A. Tsiftsis, C. C. Cavalcante, and G. K. Karagiannidis, "Multi-user and multi-relay cognitive radio networks under spectrum sharing constraints," IEEE Trans. Veh. Technol., vol. 63, no. 1, pp. 433-439, Jan. 2014.

[9] Z. Zhao, Z. Ding, M. Peng, W. Wang, and J. Thompson, "On the design of cognitive radio inspired asymmetric network coding transmissions in MIMO systems," IEEE Trans. Veh. Technol., vol. 64, no. 3, pp. 1014-1025, Mar. 2015.

[10] E. J. Leonardo and M. D. Yacoub, "The product of two $\alpha-\mu$ variates and the composite $\alpha-\mu$ multipath-shadowing model," IEEE Trans. Veh. Technol., vol. 64, no. 6, pp. 2720-2725, Jun. 2015.

[11] N. I. Miridakis, "On the ergodic capacity of underlay cognitive dual-hop AF relayed systems under non-identical generalized $-K$ fading channels," IEEE Commun. Lett., vol. 19, no. 11, pp. 1965-1968, Nov. 2015.

[12] G. Ding, J. Wang, Q. Wu, Y.-D. Yao, F. Song, and T. A. Tsiftsis, "Cellular-base-station assisted device-to-device communications in TV white space," IEEE J. Sel. Areas Commun., vol. 43, no. 1, pp. 107-121, Jan. 2016.

[13] I. F. Akyildiz, W. Y. Lee, M. C. Vuran, and S. Mohanty, "Next generation/dynamic spectrum access/cognitive radio wireless networks: a survey," Comput. Netw. J., vol. 50, no. 13, pp. 2127-2159, 2006.

[14] D. Čabrić, S. M. Mishra, and R. W. Brodersen, "Implementation issues in spectrum sensing for cognitive radios," in Proc. IEEE Conf. Signals, Syst. Comput., Nov. 2004, pp. 772-776.

[15] A. Ghasemi and E. S. Sousa, "Collaborative spectrum sensing for opportunistic access in fading environments," in Proc. IEEE 1st Int. Symp. New Frontiers Dynamic Spectrum Access Netw., Nov. 2005, pp. 131-136.

[16] P. D. Sutton, K. E. Nolan, and L. E. Doyle, "Cyclostationary signatures in practical cognitive radio applications," IEEE J. Sel. Areas Commun., vol. 26, no. 1, pp. 13-24, Jun. 2008.

[17] R. Tandra and A. Sahai, "SNR walls for signal detection," IEEE J. Sel. Topics Signal Process, vol. 2, no. 1, pp. 4-17, Feb. 2008.

[18] A. Mariani, A. Giorgetti, and M. Chiani, "Effects of noise power estimation on energy detection for cognitive radio applications," IEEE Trans. Commun., vol. 59, no. 12, pp. 3410-3420, Dec. 2011.

[19] K. J. Kim, L. Wang, T. Q. Duong, M. Elkashlan, and V. Poor, "Cognitive single carrier systems: Joint impact of multiple licensed transceivers," IEEE Trans. Wireless Commun., vol. 13, no. 12, pp. 6741-6745, Dec. 2014.

[20] P. L. Yeoh, M. Elkashlan, T. Q. Duong, N. Yang, and D. B. da Costa, "Transmit antenna selection for interference management in cognitive relay networks," IEEE Trans. Veh. Technol., vol. 63, no. 7, pp. 32503262, Sep. 2014.

[21] Y. Liu, L. Wang, T. Duy, M. Elkashlan, and T. Q. Duong, "Relay selection for security enhancement in cognitive relay networks," IEEE Wireless Commun. Lett., vol. 4, no. 1, pp. 46-49, Jan. 2015.

[22] M. Elkashlan, L. Wang, T. Q. Duong, G. K. Karagiannidis, and A. Nallanathan, "On the security of cognitive radio networks," IEEE Trans. Veh. Technol., vol. 64, no. 8, pp. 3790-3795, Aug. 2015.

[23] S. Maleki, A. Pandharipande, and G. Leus, "Energy-efficient distributed spectrum sensing for cognitive sensor networks," IEEE Sensors J., vol. 11, no. 3, pp. 565-573, Mar. 2011.

[24] Y. Deng, M. Elkashlan, N. Yang, P. L. Yeoh, and R. K. Mallik, "Impact of primary network on secondary network with generalized selection combining," IEEE Trans. Veh. Technol., vol. 64, no. 7, pp. 3280-3285, Aug. 2014.

[25] J. P. Egan, Signal Detection Theory and ROC Analysis. New York, NY, USA: Academic, 1975.
[26] H. Urkowitz, "Energy detection of unknown deterministic signals," Proc. IEEE, vol. 55, no. 4, pp. 523-531, Apr. 1967.

[27] A. Shahini, A. Bagheri, and A. Shahzadi, "A unified approach to performance analysis of energy detection with diversity receivers over Nakagami $-m$ fading channels," in Proc. IEEE Int. Conf. Connected Veh. Expo., Dec. 2013, pp. 707-712.

[28] S. Atapattu, C. Tellambura, and H. Jiang, "Energy detection of primary signals over $\eta-\mu$ fading channels," in Proc. IEEE 7th Int. Conf. Ind. Inf. Syst., 2009, pp. 118-122.

[29] P. Sofotasios, E. Rebeiz, L. Zhang, T. A. Tsiftsis, D. Čabrić, and S. Freear, "Energy detection based spectrum sensing over $\kappa-\mu$ and extreme $\kappa-\mu$ fading channels," IEEE Trans. Veh. Technol., vol. 62, no. 3, pp. 1031-1040, Mar. 2013.

[30] S. Atapattu, C. Tellambura, and H. Jiang, "Performance of an energy detector over channels with both multipath fading and shadowing," IEEE Trans. Wireless Commun., vol. 9, no. 12, pp. 3662-3670, Dec. 2010.

[31] A. Bagheri, A. Shahini, and A. Shahzadi, "Analytical and learning-based spectrum sensing over channels with both fading and shadowing," in Proc. IEEE Int. Conf. Connected Veh. Expo., Dec. 2013, pp. 699-706.

[32] S. Atapattu, C. Tellambura, and H. Jiang, "Analysis of area under the ROC curve of energy detection," IEEE Trans. Wireless Commun., vol. 9, no. 3, pp. 1216-1225, Mar. 2010.

[33] S. Atapattu, C. Tellambura, and H. Jiang, "Performance of energy detection: A complementary AUC approach," Proc. IEEE Global Telecommun. Conf., Dec. 2010, pp. 1-5.

[34] S. Alam, O. Odejide, O. Olabiyi, and A. Annamalai, "Further results on area under the ROC curve of energy detectors over generalized fading channels," in Proc. 34th IEEE Sarnoff Symp., Princeton, NJ, USA, May 2011, pp. 1-6.

[35] O. Olabiyi, S. Alam, O. Odejide, and A. Annamalai, "Efficient evaluation of area under the ROC curve of energy detectors over fading channels," in Proc. 14th ACM Int. Conf. Modeling, Anal. Simul. Wireless Mobile Syst., Miami Beach, FL, USA, Oct. 2011, pp. 261-264.

[36] S. Alam, O. Olabiyi, O. Odejide, and A. Annamalai, "A performance study of energy detection for dual-hop transmission with fixed gain relays: Area under the ROC Curve (AUC) approach," in Proc. IEEE 22nd Int. Symp. Personal Indoor Mobile Radio Commun., Sep. 2011, pp. 18401844.

[37] S. P. Herath, N. Rajatheva, and C. Tellambura, "Energy detection of unknown signals in fading and diversity reception," IEEE Trans. Commun., vol. 59, no. 9, pp. 2443-2453, Sep. 2011.

[38] S. Atapattu, C. Tellambura, and H. Jiang, "MGF based analysis of area under the ROC curve in energy detection," IEEE Commun. Lett., vol. 15, no. 12, pp. 1301-1303, Dec. 2011.

[39] A. Singh, M. R. Bhatnagar, and R. K. Mallik, "Cooperative spectrum sensing in multiple antenna based cognitive radio network using an improved energy detector," IEEE Commun. Lett., vol. 16, no. 1, pp. 64-67, Jan. 2012.

[40] S. Dikmese, P. C. Sofotasios, T. Ihalainen, M. Renfors, and M. Valkama, "Efficient energy detection methods for spectrum sensing under non-flat spectral characteristics," IEEE J. Sel. Areas Commun., vol. 33, no. 5, pp. 755-770, May 2015.

[41] S. Alam, O. Olabiyi, O. Odejide, and A. Annamalai, "A performance study of energy detection for dual-hop transmission with fixed gain relays: Area under the ROC Curve (AUC) approach," in Proc. IEEE 22nd Int. Symp. Personal Indoor Mobile Radio Commun., Sep. 2011, pp. 18401844.

[42] M. D. Yacoub, "The $\kappa-\mu$ distribution and the $\eta-\mu$ distribution," IEEE Antennas Propag. Mag., vol. 49, no. 1, pp. 68-81, Feb. 2007.

[43] M. D. Yacoub, "The $\alpha-\mu$ distribution: A physical fading model for the Stacy distribution," IEEE Trans. Veh. Technol., vol. 56, no. 1, pp. 27-34, Jan. 2007.

[44] J. F. Paris, "Statistical characterization of $\kappa-\mu$ shadowed fading," IEEE Trans. Veh. Technol., vol. 63, no. 2, pp. 518-526, Feb. 2014.

[45] M. R. Bhatnagar, "On the sum of correlated squared $\kappa-\mu$ shadowed random variables and its application to performance analysis of MRC," IEEE Trans. Veh. Technol., vol. 64, no. 6, pp. 2678-2684, Jun. 2015.

[46] G. S. Rabelo, U. S. Dias, and M. D. Yacoub, "The $\kappa-\mu$ extreme distribution," IEEE Trans. Commun., vol. 59, no. 10, pp. 2776-2785, Oct. 2011.

[47] J. I. Marcum, "Table of $Q$ functions," Rand Croporation, Santa Monica, CA, U.S. Air Force Project RAND Research Memorandum M-339, ASTIA Document AD 1165451, Jan. 1950.

[48] I. S. Gradshteyn and I. M. Ryzhik, Table of Integrals, Series, and Products, in 7th ed. New York, NY, USA: Academic, 2007. 
[49] N. Y. Ermolova, "Moment generating functions of the generalized $\eta-\mu$ and $\kappa-\mu$ distributions and their applications to performance evaluations of communication systems," IEEE Commun. Lett., vol. 12, no. 7, pp. 502504, Jul. 2008.

[50] A. M. Magableh and M, M. Matalgah, "Moment generating function of the generalized $\alpha-\mu$ distribution with applications," IEEE Commun. Lett., vol. 13, no. 6, pp. 411-413, Jun. 2009.

[51] M. Abramowitz and I. A. Stegun, Handbook of Mathematical Functions with Formulas, Graphs, and Mathematical Tables. New York, NY, USA: Dover, 1974.

[52] V. S. Adamchik and O. I. Marichev, "The algorithm for calculating integrals of hypergeometric type functions and its realization in reduce system," in Proc. Int. Symp. Symb. Algebr. Comput., 1990, pp. 212-224.

[53] A. Annamalai, C. Tellambura, and V. K. Bhargava, "Simple and accurate methods for outage analysis in cellular mobile radio systems-A unified approach," IEEE Trans. Commun., vol. 49, no. 2, pp. 303-316, Feb. 2001.

[54] J. M. Romero-Jerez and A. J. Goldsmith, "Receive antenna array strategies in fading and interference: An outage probability comparison," IEEE Trans. Wireless Commun., vol. 7, no. 3, pp. 920-932, Mar. 2008.

[55] M. Di Renzo, F. Graziosi, and F. Santucci, "Channel capacity over generalized fading channels: A novel MGF-based approach for performance analysis and design of wireless communication systems," IEEE Trans. Veh. Technol., vol. 59, no. 1, pp. 127-149, Jan. 2010.

[56] F. Yilmaz and M-S Alouini, "A unified MGF-based capacity analysis of diversity combiners over generalized fading channels," IEEE Trans. Commun., vol. 60, no. 3, pp. 862-875, Dec. 2012.

[57] H.-N. Huang, S. A. M. Marcantognini, and N. J. Young, "Chain rules for higher derivatives," The Math. Intell., vol. 28, no. 2, pp. 61-69, 2006.

[58] E. E. Fitchard and V. Franco, "Differential properties of Meijer's G-function," J. Phys. A, Math. Gen., vol. 13, no. 7, pp. 23-31, Jul. 1980.

[59] Y.-C. Ko, M.-S. Alouini, and M. K. Simon, "Analysis and optimization of switched diversity systems," IEEE Trans. Veh. Technol., vol. 49, no. 5, pp. 1813-1831, Sep. 2000.

[60] P. C. Sofotasios, T. A. Tsiftsis, Y. A. Brychkov, S. Freear, M. Valkama, and G. K. Karagiannidis, "Analytic expressions and bounds for special functions and applications in communication theory," IEEE Trans. Inf. Theory, vol. 60, no. 12, pp. 7798-7823, Dec. 2014

[61] Wolfram, The Wolfram Functions Site, [Online]. Available: http:// functions.wolfram.com

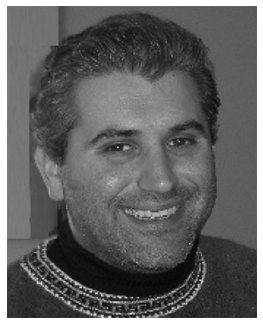

Paschalis C. Sofotasios (S'07-M'12-SM'16) was born in Volos, Greece, in 1978. He received the M.Eng. degree from Newcastle University, U.K., in 2004, the M.Sc. degree from the University of Surrey, Guildford, U.K., in 2006, and the Ph.D. degree from the University of Leeds, U.K., in 2011. His M.Sc. studies were funded by a scholarship from UKEPSRC and his Doctoral studies were sponsored by UK-EPSRC and Pace PLC. He has held academic positions at the University of Leeds, U.K., the University of California, Los Angleles, USA, Tampere University of Technology, Finland, Khalifa University, UAE, and the Aristotle University of Thessaloniki, Greece. His research interests include the broad areas of digital and optical wireless communications as well as in topics relating to special functions and statistics.

Dr. Sofotasios serves as a Regular Reviewer for several international journals and has been a Member of the Technical Program Committee of numerous IEEE conferences. He currently serves as an Editor of the IEEE COMMUNICATIONS LETTERS and he has received an Exemplary Reviewer Award by IEEE COMMUNICATIONS LETTERS in 2012 and by IEEE TRANSACTIONS ON COMMUNICATIONS in 2015 and 2016. He is a co-recipient of the Best Paper Award at ICUFN '13.

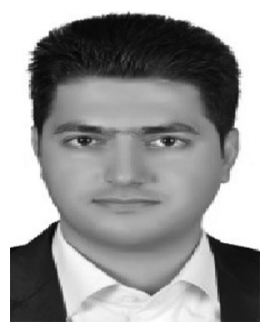

Alireza Bagheri received the M.Sc. (first-class Hons.) degree in communication systems engineering from Electrical and Computer Engineering (ECE) Department, Semnan University, Semnan, Iran, in 2013. Prior to that, he received the B.Sc. degree in electronics engineering from the ECE Department, Karaj Islamic Azad University, Karaj, Iran, in 2009. $\mathrm{He}$ is currently working toward the Ph.D. degree in electrical engineering with the Center for Wireless Information Processing, New Jersey Institute of Technology, Newark, NJ, USA. His research interests concern wireless communications, optimization, and machine learning.

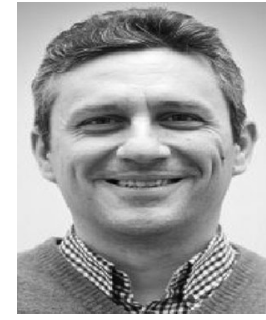

Theodoros A. Tsiftsis (S'02-M'04-SM'10) was born in Lamia, Greece, in 1970. He received the B.Sc. degree in physics from the Aristotle University of Thessaloniki, Thessaloniki, Greece, in 1993, the M.Sc. degree in digital systems engineering from the Heriot-Watt University, Edinburgh, U.K., in 1995 the M.Sc. degree in decision sciences from the Athens University of Economics and Business, Athens, Greece, in 2000, and the Ph.D. degree in electrical engineering from the University of Patras, Patras, Greece, in 2006. He joined the Department of Electrical Engineering, Technological Educational Institute of Central Greece in February 2010. He is currently an Associate Professor of Communication Technologies, School of Engineering, Nazarbayev University, Astana, Kazakhstan. He has authored or coauthored more than 120 technical papers in scientific journals and international conferences. His research interests include the broad areas of cooperative communications, cognitive radio, communication theory, wireless powered communication systems, and optical wireless communication systems.

Dr. Tsiftsis acts as Reviewer for several international journals and he was member of the Editorial Boards of IEEE TRANSACTIONS ON VEHICULAR TECHNOLOGY and IEEE COMMUNICATIONS LETTERS. He is currently an Area Editor for Wireless Communications II of the IEEE TRANSACTIONS ON COMMUNICATIONS and an Associate Editor of the IEEE TRANSACTIONS ON MoBILE COMPUTING.

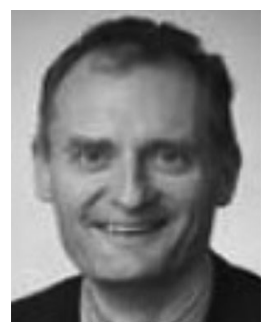

Steven Freear (S'95-M'97-SM'11) received the Doctorate degree in 1997 and subsequently worked in the electronics industry for 7 years as a ASIC System Designer. He became a Lecturer (Assistant Professor), Senior Lecturer (Associate Professor), and then a Professor in 2006, 2008, and 2016, respectively, at the School of Electronic and Electrical Engineering, University of Leeds, U.K. His main research interest is concerned with the broad area of advanced analog and digital signal processing and instrumentation. He teaches digital signal processing, VLSI and embedded systems design, and hardware description languages at both undergraduate and postgraduate levels. In June 2014, he was appointed Visiting Professor at the School of Electrical and Computer Engineering, Georgia Tech., USA, and he is External Examiner to undergraduate programmes in Electronic Engineering at Queens University, Belfast, UK.

Dr. Freear is a regular Reviewer for numerous journals and as technical programme committee member of several international conferences. He has been an Associate Editor of the IEEE TRANSACTIONS ON UlTRASOUND, FERROELECTRICS AND FREQUENCY CONTROL (T-UFFC) since 2009, and was appointed as the Editor-in-Chief in 2013.

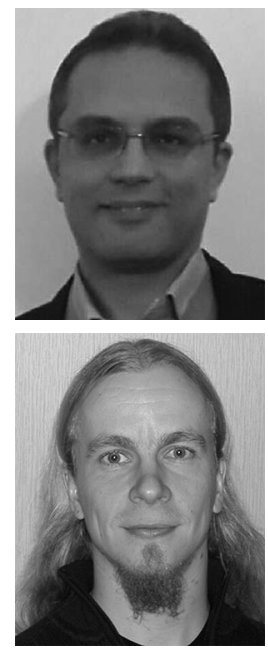

Ali Shahzadi received the B.Sc., M.Sc., and Ph.D. degrees in electrical and computer engineering from Iran University of Science and Technology, Tehran, Iran, in 1997, 2000, and 2005, respectively. He is currently an Assistant Professor of Semnan University, Semnan, Iran. His research interests include wireless, mobile, and broadband communications and networking, digital signal processing and artificial intelligence applications in communication theory.

Mikko Valkama (S'00-M'02-SM'15) was born in Pirkkala, Finland, on November 27, 1975. He received the M.Sc. and Ph.D. degrees (both with Hons.) in electrical engineering from Tampere University of Technology (TUT), Tampere, Finland, in 2000 and 2001, respectively. He received the Best Ph.D. Thesis Award by the Finnish Academy of Science and Letters for his dissertation entitled "Advanced I/Q signal processing for wideband receivers: Models and algorithms" in 2012. In 2003, he was working as a Visiting Postdoctoral Researcher with the Communications Systems and Signal Processing Institute at San Diego State University, San Diego, CA, USA. He is a Full Professor and the Laboratory Head at the Laboratory of Electronics and Communications Engineering at TUT.

His general research interests include radio communications, communications signal processing, estimation and detection techniques, signal processing algorithms for flexible radios, cognitive radio, full-duplex radio, radio localization, 5G mobile cellular radio networks, digital transmission techniques such as different variants of multicarrier modulation methods and OFDM, and radio resource management for ad-hoc and mobile networks. 